\title{
UNNOFIT inversion of spectro-polarimetric maps observed with THEMIS ${ }^{\star}$
}

\author{
V. Bommier ${ }^{1}$, E. Landi Degl'Innocenti ${ }^{2}$, M. Landolfi ${ }^{3}$, and G. Molodij ${ }^{4}$ \\ ${ }^{1}$ Laboratoire d'Étude du Rayonnement et de la Matière en Astrophysique, CNRS UMR 8112 - LERMA, Observatoire de Paris, \\ Section de Meudon, 92195 Meudon, France \\ e-mail: V.Bommier@obspm.fr \\ 2 Università degli Studi di Firenze, Dipartimento di Astronomia e Scienza dello Spazio, Largo E. Fermi 2, 50125 Firenze, Italy \\ 3 INAF-Osservatorio Astrofisico di Arcetri, Largo E. Fermi 5, 50125 Firenze, Italy \\ ${ }^{4}$ Laboratoire d'Études Spatiales et d'Instrumentation en Astrophysique, CNRS UMR 8109 - LESIA, Observatoire de Paris, \\ Section de Meudon, 92195 Meudon, France
}

Received 23 November 2005 / Accepted 8 October 2006

\section{ABSTRACT}

\begin{abstract}
Aims. We inverted a spectropolarimetric scan of an active region and a filament $(240 \times 340$ arcsec $)$ achieved with THEMIS on 7 December 2003 in the two lines Fe I 6302.5 and $6301.5 \AA$.

Methods. The inversion was achieved for each line separately by using the UNNOFIT code of Landolfi and Landi Degl'Innocenti, and was improved by introducing a magnetic filling-factor parameter. The magnetic and non-magnetic theoretical atmospheres, mixed in the proportion given by the filling factor, were derived from the same set of parameters, except for the presence (or absence) of a magnetic field. The fundamental ambiguity is not solved.

Results. The tests run with UNNOFIT show that the magnetic field strength $B$ and the magnetic filling factor $\alpha$ cannot be separately recovered by the inversion in Fe I 6302.5, but that their product $\alpha B$, which is the local average magnetic field, is recovered. The magnetic flux is only its longitudinal component. In addition, the results make two regimes clearly appear, corresponding to two ranges of local average magnetic field strength as measured in 6302.5: (a) the network, having a field inclined of about $20^{\circ}-30^{\circ}$ from the vertical in 6302.5 (spread more but non-horizontal in 6301.5), with a homogeneous azimuth. In this zone the local average field strength in 6302.5 is higher than 45 Gauss; (b) the internetwork, where the field is turbulent (with a horizontal trend, spread more at lower altitudes), and the 6302.5 local average field strength is lower than 45 Gauss (about 20 Gauss).

Conclusions. The two lines display coherent results, in particular for the magnetic-field azimuth. From this coherence we conclude that the turbulence of the 20 Gauss internetwork field has a solar origin.
\end{abstract}

Key words. Sun: magnetic fields - polarization - Sun: filament - Sun: prominences

\section{Introduction}

In his pioneering work, Stenflo (1973) retrieved information about the unresolved magnetic field of the solar photosphere from a line ratio analysis of multiline observations taken with the Kitt Peak multichannel magnetograph. He draws conclusions about an inhomogeneous structure of the photospheric magnetic field: strong fields, on the order of $2 \mathrm{kGauss,} \mathrm{would} \mathrm{be} \mathrm{concen-}$ trated in unresolved structures of about 100-300 km size, the so-called "flux tubes". Since that time, the resolution of these flux tubes has become an objective for the next instrumental progress, and in particular for building new instruments such as the THEMIS telescope. Such an objective requires simultaneous spatial, spectral and polarimetric adequate resolution. In the case of the THEMIS telescope, the adequate polarimetric resolution of $1.5 \times 10^{-3}$ is currently reached in one record on one pixel whose size is set at 0.45 arcsec, the spectral resolution being on the order of $22 \mathrm{~m} \AA$. This telescope has the original feature of being "polarization free"; i.e. the polarization analysis is performed on axis, before any oblique reflection. The second original

* Based on observations made with the French-Italian telescope THEMIS operated by the CNRS and CNR on the island of Tenerife in the Spanish Observatorio del Teide of the Instituto de Astrofísica de Canarias. feature of THEMIS is being able to simultaneously record several spectral windows, in order to probe the solar atmosphere along its depth, because the different lines simultaneously observed are formed at different altitudes. A more detailed description of the THEMIS instrument can be found in Arnaud et al. (1998), although it has to be updated with the tip-tilt correction, which has been modified and is now operational, and the polarization analyzer quarter-wave plate positions that are now free to take any position needed.

Recent results (Bommier et al. 2005b) show that it has been possible with THEMIS to scan an active region of $240 \times$ 340 arcsec size, with a pixel size of 0.45 arcsec. The data were taken on 7 December 2003, with a polarimetric accuracy allowing the analysis of all four Stokes parameters. That analysis was performed by using the bisector (or "lambdameter") method for the longitudinal field and the weak field law for the transverse magnetic field. No fractional filling factor was assumed for the magnetic field in that analysis. The objective of the present paper was to perform a second analysis of the same spectropolarimetric data, but this time submitted to an inversion code that includes a filling factor diagnostic. Before entering the details of these results, however, let us first summarize the present state of knowledge on the solar photospheric magnetic-field measurements. 
First, the line ratio technique has been pursued until recently, thus not severely modifying the result obtained by Stenflo. ZIMPOL I observations have confirmed the expected order of magnitude of 1 kGauss for field strength (Keller et al. 1994, who in addition suggest lower field strength in internetwork regions, and Grossmann-Doerth et al. 1996, who estimate that the concentration degree of the magnetic field should be high). More recently, Dominguez Cerdeña et al. (2003) estimate a field strength of about $1 \mathrm{kGauss}$ associated to a filling factor of $2 \%$ in internetwork regions $(75 \mathrm{~km}$ is the largest compatible size for a magnetic element). Similar orders of magnitude have been independently derived by Lites \& Socas Navarro (2004).

Infrared (IR) observations open direct access to the field strength determination via the measurement of the Zeeman splitting, which is more resolved in this wavelength range. Two different investigations (Lin 1995; Khomenko et al. 2003) result in evidence of two field strength ranges for network (higher strength) and internetwork regions (lower strength). A simultaneous IR and visible observation (Lin \& Rimmele 1999) confirm the low level $(1 \%)$ of the filling factor.

As suggested by these IR observations, which are highly convincing due to the direct character of the field strength measurement, models of magnetic atmosphere with two (or more) mixed components were developed: a) an SIR (Ruiz Cobo \& del Toro Iniesta 1992) inversion with two magnetic components of different strengths was performed by assigning the mixed field strength to both network and internetwork regions (Socas Navarro \& Lites 2004). Only $25 \%$ of the pixels were analyzed and strong $\mathrm{kG}$ fields found in most of the inverted spatial pixels; b) a MISMA (Sánchez Almeida 1997) inversion was applied by Sánchez Almeida \& Lites (2000), with inclusion of three components: two magnetic and one non-magnetic. Again, similar field strengths were derived for the two magnetic components. A MISMA inversion including a PCA (principal components analysis) confirms the ubiquity of $\mathrm{kG}$ field strengths even outside the network (Socas Navarro \& Sánchez Almeida 2002), in the $25 \%$ of pixels that are analyzed. However, refined observations and analysis have confirmed the visible/IR discrepancy (Sánchez Almeida et al. 2003). The inversion of visible lines (Grossman-Doerth et al. 1996; Sanchez Almeida \& Lites 2000; Socas-Navarro \& Sanchez Almeida 2002; Dominguez Cerdeña et al. 2003) leads to the conclusion that a notable fraction of fields are found in the kilogauss range, while the works with IR lines (Lin 1995; or Khomenko et al. 2003, among others) indicate that strong fields are very rare. These big discrepancies have produced a lot of papers that try to find its origin.

At this stage, it has to be pointed out that all these analyses (except the one of Khomenko et al. 2003) only involve the circular polarization Stokes parameter $V$, so that poor information is retrieved for the field direction. Though the basic SIR method includes determination of magnetic field inclination and azimuth, their determination seems not to have been included in the latest analysis that involves only the Stokes parameter $V$. In the MISMA model, the magnetic field is assumed to be vertical. A full Stokes observation and analysis has been performed by Lites (2002), who notes that the linear polarization degree is higher in the internetwork regions than in the network, so that he concludes that there are (i) a homogeneous and vertical field in the network with a higher strength and (ii) a "mixed polarities" field in the internetwork regions with a lower strength. Khomenko et al. (2003) also measured the linear polarization and concludes that "the magnetic field has a broad range of inclinations, although most of the pixels show polarization signatures that imply an inclination of about $20^{\circ}$ ".
Some of the aforementioned papers investigate in addition the relationship between the field and the granule/intergranule structure; due to the absence of a tip-tilt regulation on THEMIS at the time our data were taken, this study is beyond the scope of the present paper.

Another method of investigating the photospheric/ chromospheric magnetic field has been provided by the interpretation of the so-called "second solar spectrum" observation, which is the observation of the linear polarization due to scattering near the solar limb. This polarization may be modified due to the Hanle effect, so that information on the weak magnetic field can be retrieved in this way. As the Hanle rotation of the polarization direction has never been detected in the second spectrum of quiet region photospheric lines, while the Hanle magnetic depolarization has been observed, it was concluded that the magnetic field has a so-called "turbulent" (i.e. unresolved) direction (Stenflo 1982). Recent interpretations of Sr I $4607 \AA$ A measurements have independently led to a turbulent field strength of 35-60 G (Faurobert et al. 2001; Bommier et al. 2005a; Trujillo Bueno et al. 2004). In addition, Trujillo Bueno et al. (2004) investigate a possible PDF (probability distribution function) for this field strength. Their investigation of the scattering polarization observed in the Sr I 4607 line, based on three-dimensional radiative transfer calculations using realistic hydrodynamical photospheric models, indicates that the mean field strength of the "turbulent field" is $\sim 100 \mathrm{G}$, which is higher than in previous investigations (see, e.g., Faurobert et al. 2001). Their conclusion has been confirmed by Bommier et al. (2005a), at least for the case of a single-value microturbulent field that fills the entire photospheric volume. Moreover, a joined analysis of the Hanle effect in the Sr I 4607 line and in $\mathrm{C}_{2}$ lines (see Trujillo Bueno et al. 2004) suggests that most of this "hidden" magnetic flux is located in the intergranular regions of solar-surface convection.

The inversion code and the implementation of the fillingfactor determination are described in Sect. 2. In particular, the accuracy of the determination was investigated. The inversion was independently performed in two lines, Fe I 6302.5 and $6301.5 \AA$ A. The 6302.5 results are described in Sect. 3 , and the 6301.5 ones in Sect. 4. The comparison between the two line results is then achieved (Sect. 5), showing coherence especially in magnetic field azimuth. This coherence leads to the conclusion that the origin of the observed internetwork field turbulence is at least partly solar.

The solution of the fundamental ambiguity, which is that two field vectors that are symmetrical with respect to the line-ofsight have the same polarimetric signature, is beyond the scope of the present paper. Disambiguation has not been performed. In this respect, the orientation of the transverse field along its direction is unknown, so that the transverse field direction has been indicated by dashes without any arrow in the following maps.

Besides going further in the paper, we have to clarify what meaning we assign here to the word 'turbulent' when applied to the magnetic field. We mean a field whose direction randomly changes from one pixel to the neighboring one. No other signification is attached to this word in the present work.

\section{The UNNOFIT inversion code}

\subsection{Presentation}

This section is intented to describe the code and how the magnetic filling-factor parameter was introduced in it. 
The UNNOFIT inversion code is based on the Marquardt algorithm to reach the minimum theory/observation discrepancy with the theoretical profiles given by the Unno-Rachkowsky solution. Pionereed by Harvey et al. (1972) and Auer et al. (1977), this technique has been improved by Landolfi \& Landi Degl'Innocenti (1982) and Landolfi et al. (1984) to allow for magneto-optical and damping effects. The same Marquardt algorithm technique, based on the Unno-Rachkowsky solution that includes the magneto-optical effects as introduced by Landolfi \& Landi Degl'Innocenti (1982) has been applied to sunspot observations inversion by Skumanich \& Lites (1987) and Lites \& Skumanich (1990), who implemented additional reduction procedures in their code.

As described by its authors Landolfi et al. (1984), UNNOFIT - the straightforward application of the technique outlined above (see also Landi Degl'Innocenti \& Landolfi 2004) - provides simultaneous determination of eight free parameters via the fit of the four Stokes profiles. As in Skumanich \& Lites (1987), different weights can be assigned to the different Stokes parameters, usually 0.1 for $I$ and 1 for $Q, U, V$. The eight free parameters are 1) the line strength $\eta_{0} ; 2$ ) the Zeeman splitting $\Delta \lambda_{\mathrm{H}}$ that provides the magnetic field strength; 3 ) the Doppler absorption profile width $\left.\Delta \lambda_{\mathrm{D}} ; 4\right)$ the $\gamma$ damping parameter of the Voigt function; 5) one single $b$ parameter describing the Milne-Eddington $\tau$-dependence along the atmosphere vertical with $b=\mu B_{1} / B_{0}$, where $B_{0}$ and $B_{1}$ are the usual parameters describing the MilneEddington atmosphere, and $\mu$ is the cosine of the line-of-sight inclination angle; 6 ) the line central wavelength (providing thus the Doppler shift), 7) and 8) the magnetic field inclination and azimuth angles. The Marquardt algorithm is an iterative method of reaching the minimum of the chi-square parameter that characterizes the theory/observation discrepancy. It makes use of the partial derivatives of the functions giving the observed parameters with respect to the eight parameters to be determined. With this purpose, these functions have preferably to be analytical functions so that their derivatives can be explicitly written down. This requirement is fulfilled by the Unno-Rachkowsky solution in the Milne-Eddington atmosphere. The iteration is initialized by a random draw of the eight parameters. This random character has led to repeating the iteration for each pixel 20 times, with a different initial draw each time. The iteration is stopped when one of the three following requirements is fulfilled: a) when the number of iterations gets larger than a previously fixed number (presently 60); b) when the chi-square gets smaller than a small fraction (presently $10^{-10}$ ) of the initial chi-square (the one calculated at the beginning with random numbers); c): when the sum of the absolute values of the eight increments gets smaller than a fixed number. The final result is the one corresponding to the lowest chi-square value of the 20 iterations.

In the present work, we have added a ninth free parameter to be determined by UNNOFIT: the filling factor $\alpha$, which means that the received radiation is the sum of the magnetic component radiation, weighted $\alpha$, and of the non-magnetic component one, weighted $1-\alpha$; that is to say, denoting by " $\mathrm{m}$ " and "nm" the magnetic and non-magnetic contributions, one has

$\left\{\begin{array}{l}I=(1-\alpha) I_{\mathrm{nm}}+\alpha I_{\mathrm{m}} \\ Q=\alpha Q_{\mathrm{m}} \\ U=\alpha U_{\mathrm{m}} \\ V=\alpha V_{\mathrm{m}}\end{array}\right.$

The calculation of the derivatives with respect to the $\alpha$ parameter is straightforward, so that the implementation of this ninth parameter in the algorithm follows. The magnetic and nonmagnetic components of the atmosphere have all their physical parameters taken as equal, except the three magnetic field coordinates. In the present work, the same weight 1 was given to the four Stokes parameters for the chi-square calculation.

Note that this procedure is different from the one used by Skumanich \& Lites (1987), where the quantity $I_{\mathrm{nm}}$ of Eq. (1) is assumed to be known a-priori, being given by the average intensity profile over the observed area, excluding sunspots and active regions. Our procedure does not take into account any difference in physical conditions (temperature, density) between the internal and the external parts of the fluxtube, although the presence/absence of the magnetic field is able to modify the matter motions. However, variations in these conditions on a medium spatial scale (across plage regions, for instance) are fully taken into account by our procedure, which is not the case under the constant $I_{\mathrm{nm}}$ hypothesis of Skumanich \& Lites (1987) and which may be more realistic than a constant $I_{\mathrm{nm}}$ throughout the whole map. In any case, it has to be expected that the results on the magnetic field and filling factor, since mostly related to the polarization profiles, should be somewhat independent of the parametrization chosen for $I_{\mathrm{nm}}$. Besides, the assumption of same physical parameters inside and outside the fluxtubes is at most questionable in the sunpots and their environment. Although there are 1.5 sunspots in our scan, their detailed study is not the aim of the present paper, which is devoted to network/internetwork characterization.

The Fe I $6302.5 \AA$ line is a normal Zeeman triplet, being ${ }^{5} D_{0}-{ }^{5} P_{1}$ and having $J_{u}=0$ and $J_{\ell}=1$ with $\mathrm{g}_{\ell}=5 / 2$. The Fe I $6301.5 \AA$ line is not a normal Zeeman triplet, being ${ }^{5} D_{2}-{ }^{5} P_{2}$ (same multiplet as 6302.5, the 816 multiplet) and having $J_{u}=2$ and $J_{\ell}=2$ with $\mathrm{g}_{u}=3 / 2$ and $\mathrm{g}_{\ell}=11 / 6$. The UNNOFIT code has two versions: one for the normal Zeeman triplet line, UNNOFIT, which has been applied to 6302.5, and one for a non-triplet line taking the true Zeeman splitting into account, UNNOFIT2 that has been applied to 6301.5 (in UNNOFIT2 40 iterations are preferable). We checked that UNNOFIT and UNNOFIT2 give coherent results when applied to the same line, 6302.5. The two lines 6301.5 and 6302.5 were then independently inverted.

\subsection{Accuracy}

First, the polarimetric accuracy was investigated on 8 pixels distributed throughout the map. By applying a wavelet filtering, the average signal was subtracted leading to the possibility of measuring the noise along the polarization profile. The noise average value $1.5 \times 10^{-3}$ was thus determined, for each Stokes profile $S / I$, $S$ being one of the Stokes $Q, U$ or $V$ (24 noise values averaged). This value of $1.5 \times 10^{-3}$ is also the photon noise level.

An example of the fit result is given in Fig. 1, for a typical low-polarization internetwork pixel showing the $1.5 \times 10^{-3}$ noise level mentioned. Figures 2 and 3 represent, for the same pixel, the variation in the $\chi$ parameter of the fit with two parameters of the model: a) the magnetic field strength and the filling factor for Fig. 2a, and b) the field inclination and azimuth angles for Fig. 3, where the other parameters are taken at their $\chi^{2}$-minimum value. No secondary minimum appears on the surfaces, in particular in the high filling factor range (recall that the azimuth is defined modulo $180^{\circ}$ ). The full-color scale is determined by the noise level $1.5 \times 10^{-3}$ divided by the square root of the pixel number involved in the $\chi^{2}$ calculation.

Note that this level is not far above the $10^{-3}$ limit level determined by Bellot Rubio \& Collados (2003) for correct interpretation of the Fe I visible line polarization. Moreover, with respect 


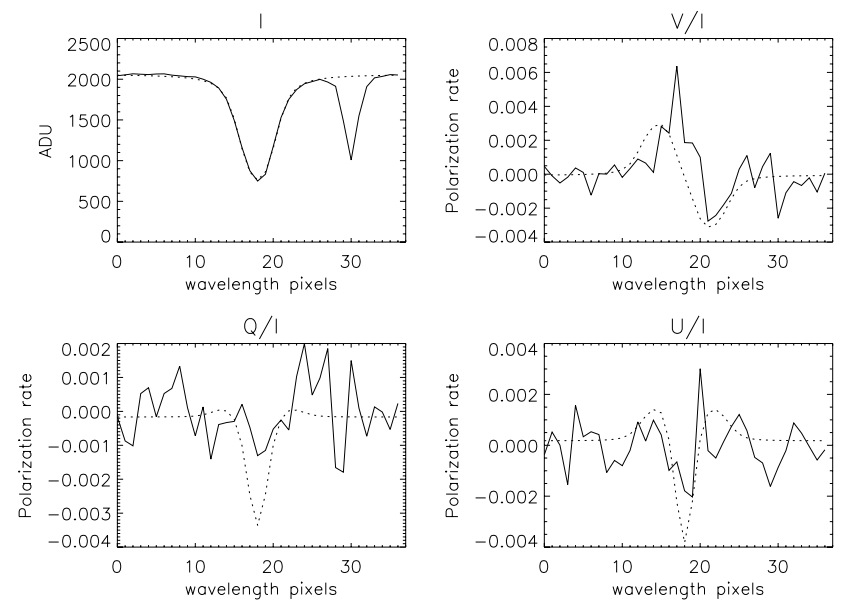

Fig. 1. Fit for a typical low polarization (internetwork) pixel (pixel 151,151) of the 7 December 2003 map. The central line is Fe I $6302.5 \AA$, adjacent to a telluric line. Full line: observed intensity and polarization profiles. Dotted line: UNNOFIT result. The obtained magnetic field strength is 1619 Gauss and the magnetic filling factor $\alpha=0.012$, leading to the significant local average magnetic field strength $\alpha B=$ 19 Gauss. The field inclination is $52^{\circ}$ and the azimuth is $36^{\circ}$ with respect to the slit direction. The wavelength pixels $28-33$ that correspond to the telluric line have been discarded from the fitting procedure. The chisquare value, summed over the four Stokes parameters and the 31 analyzed wavelength pixels, is 0.0021 in polarization units (i.e., all the Stokes parameters having been divided by the continuum intensity).

to the work of these authors, it has to be emphasized that we independently performed the two Fe I-line inversion (whereas they treat both lines within the same atmosphere, although 6302.5 is formed $66 \mathrm{~km}$ lower than 6301.5) and that we made use of both linear and circular polarization in the inversion (whereas they consider Stokes $V$ only). However Figs. 2 and 3 do not really provide the accuracy of the field-vector determination: they only prove the uniqueness of the minimum.

Then to investigate the inversion accuracy, we proceeded in a pragmatic manner. Given a series of 183600 field and filling factor values (field strength ranging from 100 to 3000 Gauss with 100 Gauss steps, field inclination ranging from $10^{\circ}$ to $170^{\circ}$, and azimuth ranging from $0^{\circ}$ to $170^{\circ}$, both with $10^{\circ}$ steps, 20 filling-factor values ranging in a logarithmic scale between 0.01 and $<1$ ), we computed the theoretical profiles that would result from these fields, by applying the Unno-Rachkowsky solution. We then added a noise to these theoretical profiles. The noise that we used was not Gaussian, a random number taken between $-3 \times 10^{-3}$ and $+3 \times 10^{-3}$ (given the noise level $1.5 \times 10^{-3}$ in the observed profiles). We submitted these noised theoretical profiles to the UNNOFIT inversion, and we then compared the obtained magnetic fields ("output") with the initial ones ("input").

We did that first for the Fe I 6302.5 line (UNNOFIT code). The first result (Fig. 4) is that, although the magnetic field strength $B$ and magnetic filling factor $\alpha$ are not separately recovered by the inversion (see the first row of the figure where a wide range of output values correspond to each given input value), their product $\alpha B$ is recovered (see the second row of the figure giving a sufficient alignment on the diagonal). The value of $\alpha B$ is the local average magnetic field strength, and the magnetic flux is only the longitudinal component of the corresponding vector. This behavior is easily explained by how, in weak fields, the spectral behavior of the Stokes profile $V / I$ is not determined by the field strength, but by the first derivative of the intensity profile, whereas the field strength $B$ and the magnetic filling factor $\alpha$ both act in the same manner on the $V / I$ magnitude, so that they cannot be distinguished. As the present method only permits the determination of the local average field $\alpha B$, we will consider this quantity (together with the field direction) instead of $\alpha$ and $B$ separately in the following.

At this stage, it has to be pointed out that the determination of the average local magnetic field can only be done with the present form of UNNOFIT that was completed with the filling factor determination: our tests show that forcing $\alpha$ to remain at unity does not lead to the correct local average magnetic field determination.

Looking at the lowest values in the bottom right figure of Fig. 4, it can be seen that input values of the local average magnetic field lower than 5 Gauss are not recovered in the output, which gives them the mean value of 20 Gauss instead. In other words, if a 20 Gauss local average field is determined by the inversion, it may also be a local average field of a few Gauss. This point is of some importance for further discussions.

The histograms of the differences "output" minus "input" are displayed in Fig. 5, where we have separated the input values $\alpha B \geq 45$ Gauss from the input values $\alpha B<45$ Gauss, which later correspond to network and internetwork values, respectively. We put the histogram widths in the same category as the UNNOFIT accuracy under our observation conditions: in the network, the local average magnetic field strength $\alpha B$ is obtained \pm 5 Gauss, and the inclination and azimuth angles of the field vector are obtained $\pm 5^{\circ}$. In the internetwork, the fact that lower values of $\alpha B$ may be confused with 20 Gauss values is clearly visible in the histogram, which is not centered on 0 but on 10 Gauss, with a \pm 5 Gauss width. The inclination and azimuth angles of the field vector are obtained $\pm 20^{\circ}$. Plotting the azimuth histograms for $\alpha B<10$ Gauss and $\alpha B<5$ Gauss shows that the azimuths are fully indeterminate (flat histogram) for $\alpha B<5$ Gauss, so that the inaccuracy in field strength is 5 Gauss, given the frequency sampling and the polarimetric inaccuracy taken from the measurements. Five Gauss can be considered as the magnetic field accuracy of the present measurements, and was also, in accordance, the accuracy of the previous longitudinal field measurements via the "lambdameter" method (Bommier et al. 2005b). For $\alpha B<10$ Gauss, the histograms are not completely flat, leading to some angle determination by the inversion even in this case, though with a larger uncertainty.

Figures 6 and 7 display the same kind of results, but now for the Fe I 6301.5 line (UNNOFIT2 code). The 6301.5 line is less sensitive to the Zeeman effect than is 6302. This lower sensitivity is visible in the results, where the trends are, however, the same, as it is impossible to determine separately the field strength $B$ and the magnetic filling factor $\alpha$. Only the local average magnetic field strength $\alpha B$ can be determined. In the network, the field direction is determined within a $\pm 10^{\circ}$ accuracy, whereas in the internetwork the accuracy on the field direction determination is $\pm 30^{\circ}$. For Fe I 6301.5, the inaccuracy in field strength is higher than with Fe I 6302.5. This inaccuracy is 10 Gauss, with a flat azimuth histogram when the values $\alpha B<10$ Gauss are selected. The histogram is not completely flat, leading to an angle determination by the inversion, when the values $\alpha B<20$ Gauss are selected.

Besides this, the accuracy of the Milne-Eddington approximation was tested by inverting profiles obtained from a nonLTE solution based on the integral equation for the atomic density matrix elements in an arbitrary magnetic field (see the theory in Landi Degl'Innocenti et al. 1991a,b, and the numerical application in Bommier \& Landi Degl'Innocenti 1996). This solution was particularized to the case of a Quiet Sun 

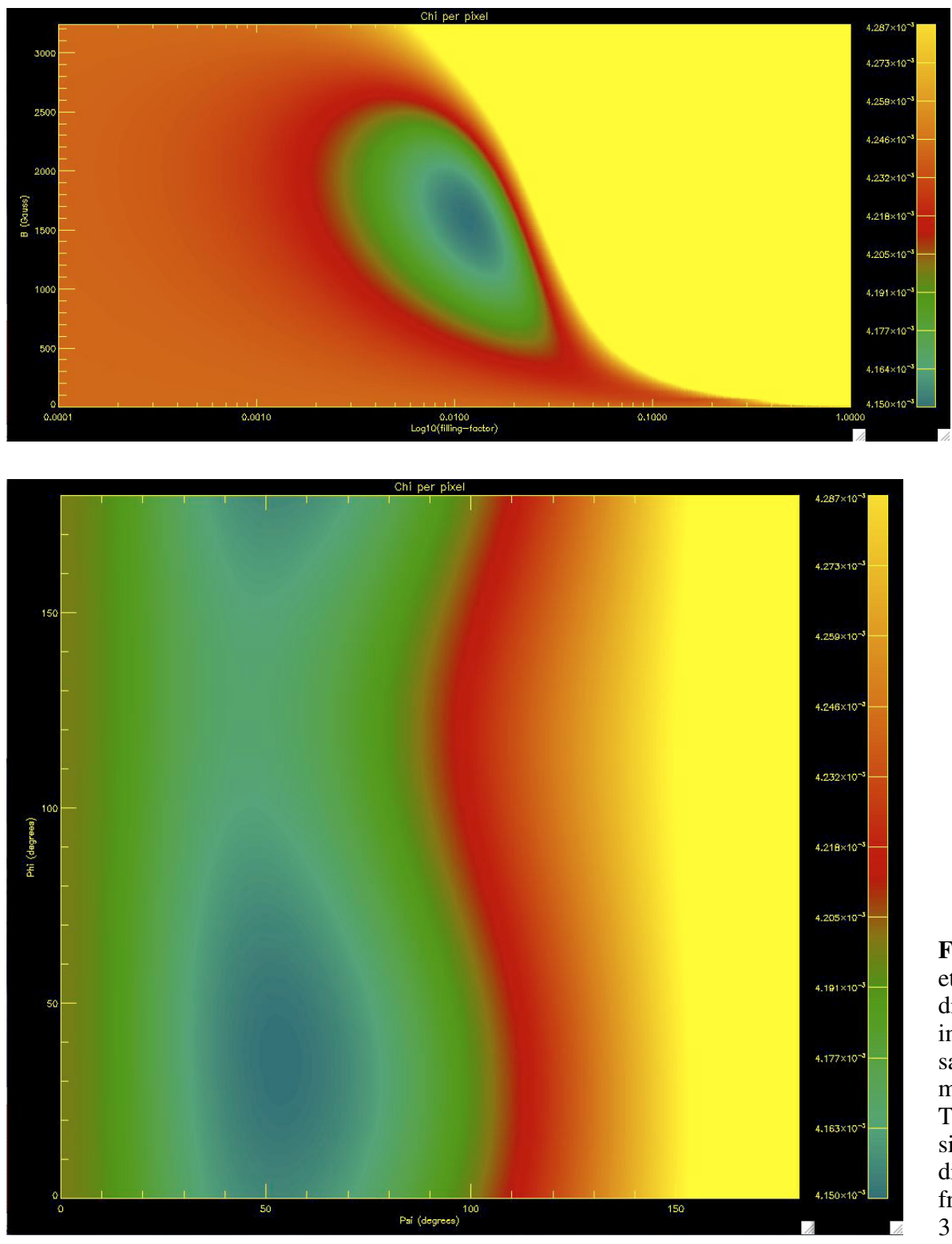

Fig. 2. Minimum of $\chi$ for two varying parameters: the magnetic filling factor (in abscissae, logarithmic scale) and the magnetic field strength (in ordonnae), for the same pixel as in Fig. 1. The other parameters of the model have been taken at their $\chi^{2}$-minimum value. The color scale has been limited to an $\chi$ variation inside the polarimetric sensitivity per pixel, 0.0015 , divided by the square root of the total number of frequency pixels entering the $\chi$ calculation, namely $31 \times 4$ pixels.

Fig. 3. Minimum of $\chi$ for two other varying parameters that are the two angles defining the field vector direction: the inclination (in abscissae), and the azimuth (in ordonnae, defined modulo $180^{\circ}$ ), for the same pixel as in Fig. 2. The other parameters of the model have been taken at their $\chi^{2}$-minimum value. The color scale was limited to an $\chi$ variation inside the polarimetric sensitivity per pixel, 0.0015 , divided by the square root of the total number of frequency pixels entering the $\chi$ calculation, namely $31 \times 4$ pixels.

atmosphere model, following the method described in Bommier et al. (2005a) for the Sr I 4607 line, presently extended to the Fe I 6302.5 line for the line opacity calculation (see also Sect. 4). The source function in zero magnetic field is given in Fig. 8. As the field-free approximation is valid in a homogeneous atmosphere (Rees 1969; see also Trujillo Bueno \& Landi Degl'Innocenti 1996), we verified that the source function in non-zero magnetic field is close to the zero magnetic field one. The UNNOFIT solution was computed for several field directions, the field strength being taken as 1500 Gauss (network) and 500 Gauss (internetwork) and the magnetic filling factor as 0.05 . In all cases the UNNOFIT solution was found to discard no more than $10^{\circ}$ from the input values for the inclination and azimuth angles and no more than about 10 Gauss for the local average field strength $\alpha B$. It can be seen in Fig. 8 (lower figure) that the behavior of the source function is linear in the low $\tau$ region, as is the case of the Milne-Eddington approximation, which explains that the output field agrees with the input one. A larger departure from the Milne-Eddington approximation can, however, be expected in the plages and even more in the sunspot, neither of which the main subject of investigation in the present work.

\subsection{Symmetrization of the polarization profiles}

In this fit of observed profiles, the question arises about their symmetries, which are broken in particular due to the velocity effects. We used a correction for these velocity effects, as follows:

- Since $Q$ and $U$ are symmetrical profiles, all the $I+Q, I-Q, I+$ $U$, and $I-U$ profiles are displaced in frequency by the radial velocity only and not by the magnetic field. The correction consists then in recentering all the individual profiles $I+Q$, $I-Q, I+U$, and $I-U$ at each of the two times of the beam exchange before combining them for extracting $Q / I$ and $U / I$. This assumes that a line position method is used in the data analysis. We used the one of Bommier \& Rayrole (2002).

- The situation is more complex with $V$, because $I+V$ and $I-V$ are displaced in frequency by both the radial velocity and the magnetic field. However, something can be done to take advantage of the beam exchange. By summing the $I+V$ and $I-V$ images obtained in the same channel at the different times of the beam exchange, one obtains a single $I$ profile 

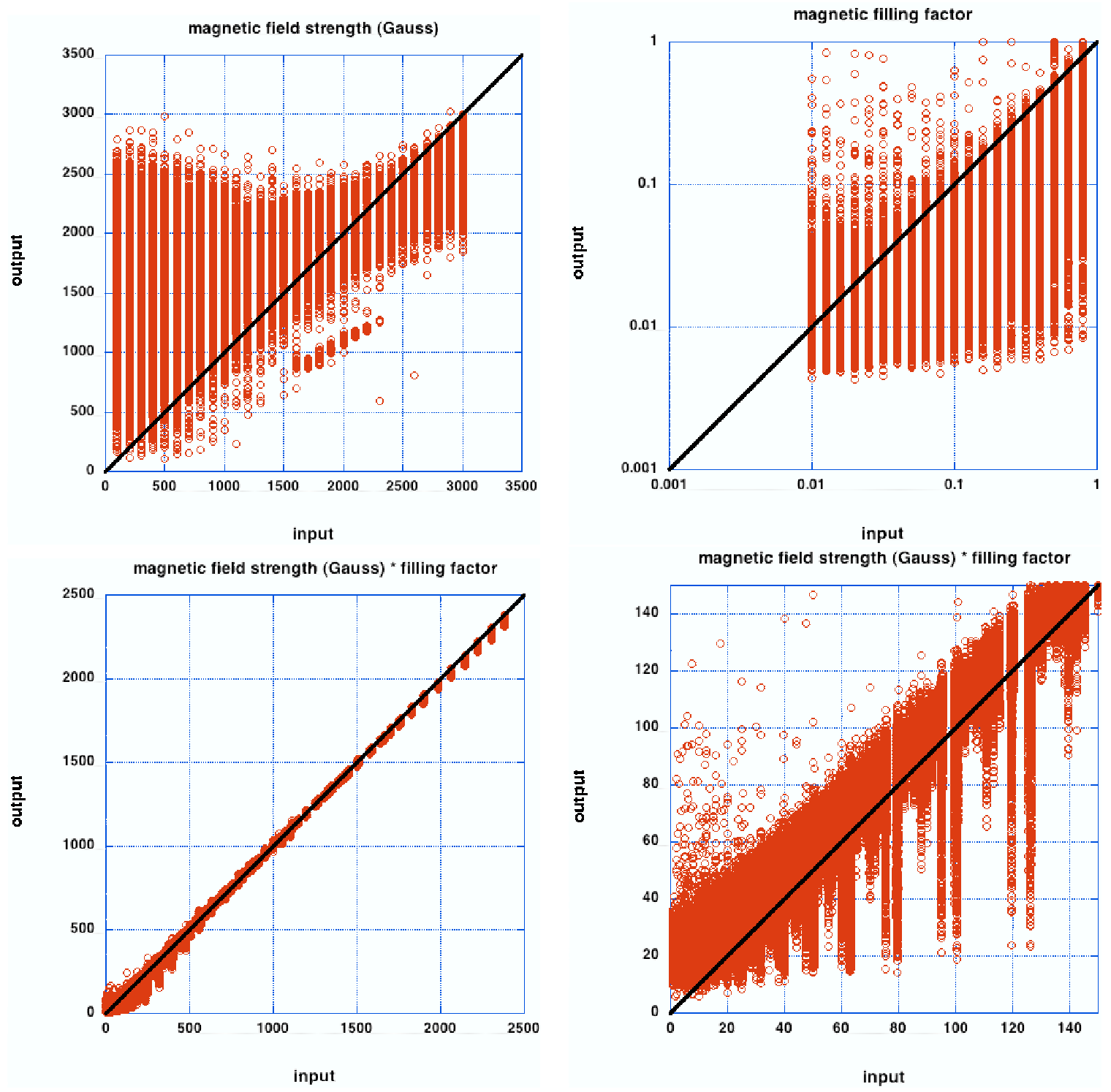

Fig. 4. Comparison between the UNNOFIT output values with the input ones given as a series of values in a file (see text) for the line Fe I 6302.5 . For each ensemble of input values (abscissae), a set of theoretical profiles has been computed, noised, and then inverted, leading to the output values (ordonnae). Upper row: left: magnetic field strength; right: magnetic filling factor. Lower row: local average magnetic field strength, which is the product of the magnetic field strength by the magnetic filling factor, the right figure being a zoom on the left one. The figure demonstrates that, although the magnetic field strength and magnetic filling factor are not separately recovered by the inversion, their product is recovered.

displaced only by the radial velocity. This provides the shift needed to apply to all the profiles observed in the channel under study, to correct for the radial velocity effects. The shift is then applied to all the individual profiles $I+V$ and $I-V$ of each of the two beams before combining them for extracting $V / I$.

This procedure is called "symmetrization" here because its effect is to render the profiles more symmetric (for $Q / I$ and $U / I$ ) or antisymmetric (for $V / I$ ). In practice, it is only a correction of the radial velocity change between the two exposures required by the beam exchange technique.

\section{Results from Fe I $6302.5 \AA$}

Figure 9 displays the $\mathrm{H} \alpha$ image of a filament lying in the image center, and one of the two spots of the NOAA 517 active region in the upper part of the image (half of the second spot is visible on the left edge). Figure 10 displays the magnetic field solution of the UNNOFIT procedure. Although derived in terms of local average field strength, inclination, and azimuth, the field vector is drawn in terms of longitudinal (in colors) and transverse (in dashes) components. These components are expressed in the line-of-sight and plane of the sky coordinates, but they are not very different from the solar coordinates because the filament is located near the disk center $(15 \mathrm{~S}-15 \mathrm{~W})$. It appears on this map that the regions with the strongest longitudinal component also show a homogeneous transverse field in both intensity and azimuth. These regions draw the network. In contrast to the homogenous shape of the network field, the internetwork field, although too small to be visible in the figure, appears to be fully "turbulent", i.e. its direction changes from one pixel to the next.

These two kinds of regions also correspond to a local average field strength larger or smaller than 45 Gauss. The contour of the local average field strength 45 Gauss was drawn in the figure above the $\mathrm{H} \alpha$ image (bottom layer). It appears that 


\section{$\alpha B$ (input 6302) $>=45$ NETWORK}
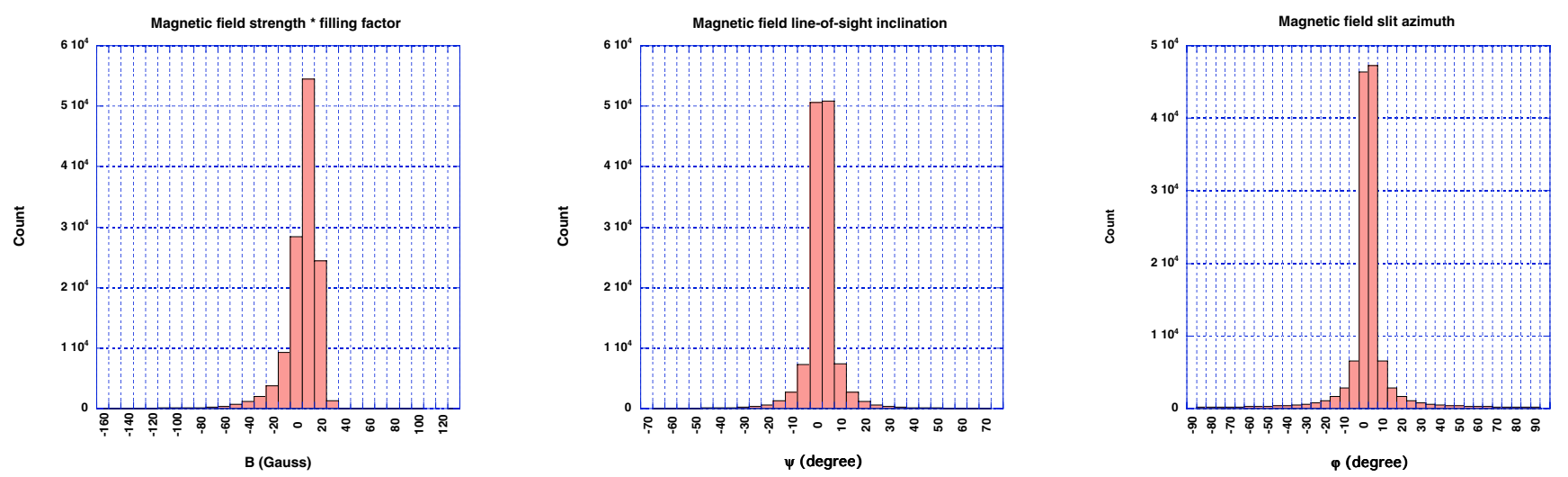

\section{$\alpha B$ (input 6302) $<45$ INTERNETWORK}
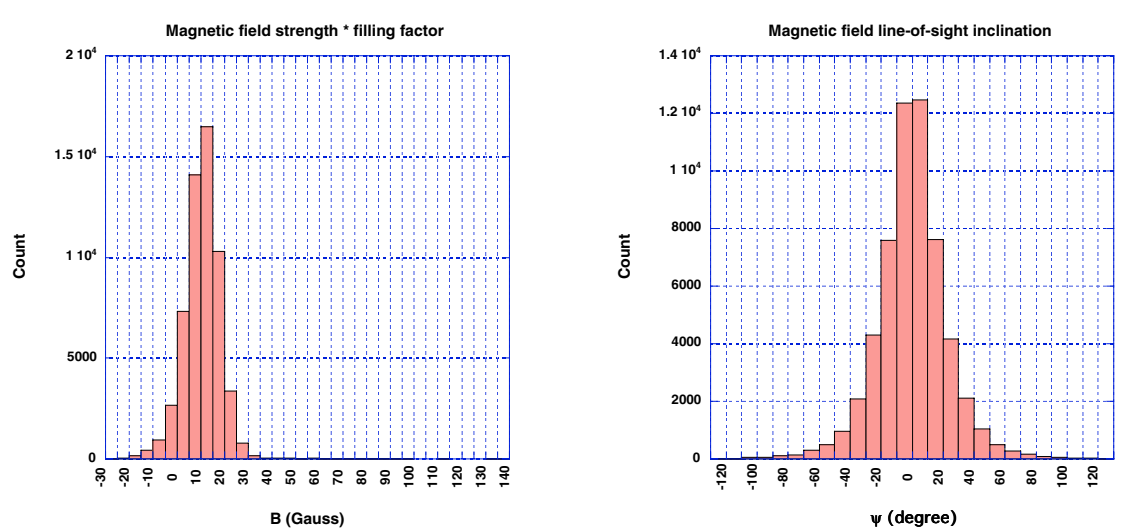

Fig. 5. Accuracy of the UNNOFIT inversion (Fe I 6302.5 line): histograms of the differences between the UNNOFIT output values and the input ones given as a series of values in a file (see text). For each ensemble of input values, a set of theoretical profiles has been computed, noised, and then inverted, leading to the output values. Histograms have been plotted for $1 /$ the local average magnetic field strength, which is the product of the magnetic field strength by the magnetic filling factor, 2 / the line-of-sight inclination angle, and 3/ the slit azimuth angle. The histograms have been plotted for local average magnetic field strength higher and lower than 45 Gauss separately, corresponding on the maps to network and internetwork values, respectively.

these contours draw the network as the higher local average field strength regions. By comparing with Fig. 9, it is also visible that these network regions have stronger $\mathrm{H} \alpha$ emission so that they are plages. The filament separates two regions where the network has opposite polarity, and just below the filament the field appears to be of the internetwork type, namely turbulent. Figure 12 displays the map of the magnetic field line-of-sight inclination angle (angle between the field vector and the line-of-sight): the vertical trend in the network and the horizontal trend internetwork are visible due to their different colors. The contour of the $\mathrm{H} \alpha$ filament has been superimposed, to facilitate studying the relationship between the filament and the polarities.

The difference between the two kinds of region is made evident by the histograms plotted in Fig. 13 for local average field strength larger or smaller than 45 Gauss. The upper row of the figure that is associated to local average field strength $\alpha B$ larger than 45 Gauss displays a regularly decreasing histogram for the magnetic field strength. In this class of pixels, the inclination histogram is strongly peaked at $25^{\circ}$ and $155^{\circ}$, this last case corresponding to the same inclination with opposite polarity. A small secondary peak at the horizontal field (inclination $90^{\circ}$ ) appears in the upper part of the map in the sunspot penumbra. The azimuth histogram shows a prominent peak, so that the magnetic field vector found in this region turns out to be very homogeneous in strength and direction (both inclination and azimuth). As for the histograms in the lower part of the figure that are associated to local average field strength $\alpha B$ smaller than 45 Gauss, the local average field strength histogram shows a maximum around $\alpha B=20$ Gauss. As pointed out in Sect. 2.2, this value of 20 Gauss may eventually be compatible with the existence of weaker fields. The direction histograms allow nearly all possible directions, thus giving the image of a turbulent field: the azimuth 

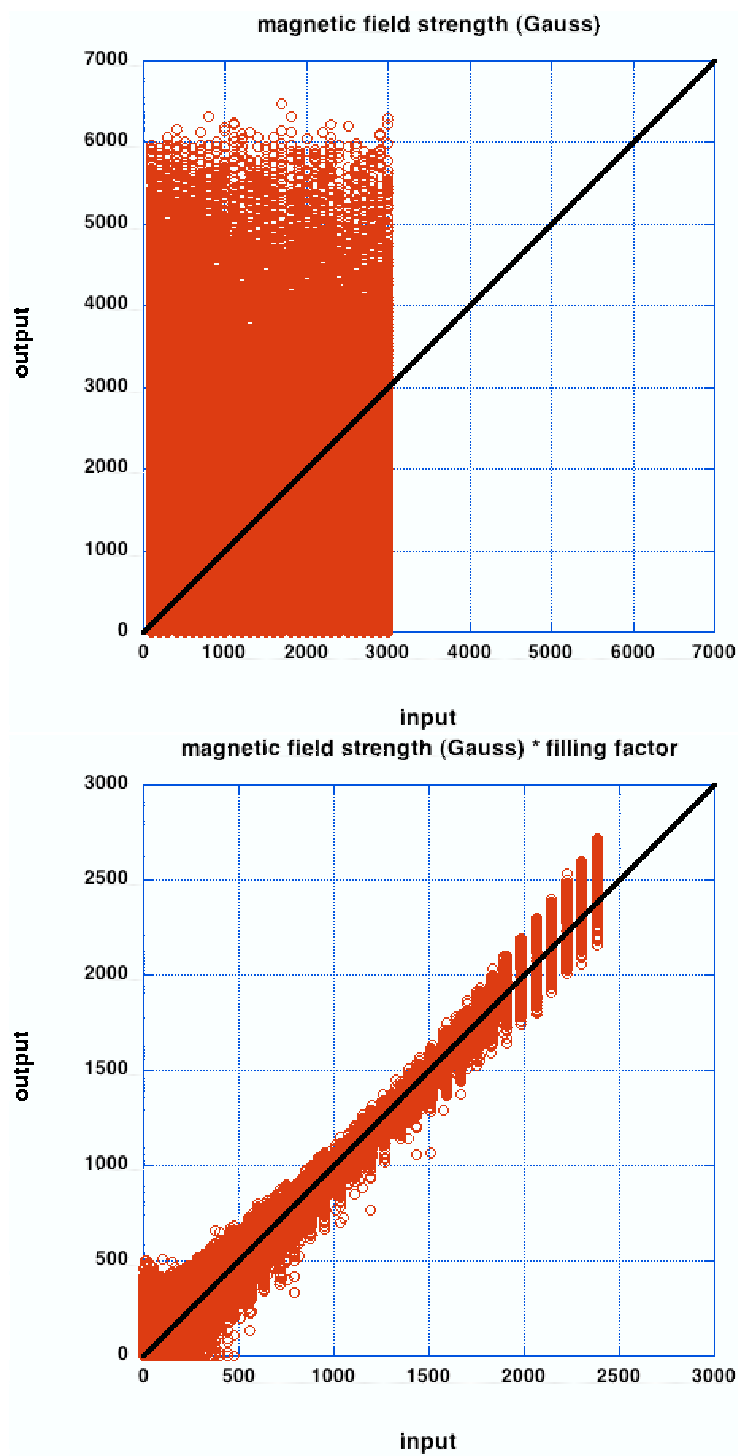

Fig. 6. Same as Fig. 4, but now for the Fe I 6301.5 line.

is random, but the inclination remains more or less horizontal, contained between $35^{\circ}$ and $145^{\circ}$. These histograms then lead to the following conclusion. The value $\alpha B=45$ Gauss separates two very different regimes: a) $\alpha B>45$ Gauss corresponds to the network. In this regime, the field direction is homogeneous and inclined $25^{\circ}$ (not too far) from the vertical. b) $\alpha B<45$ Gauss corresponds to the internetwork regions where the field is fully turbulent in direction, showing nevertheless a more or less horizontal trend with inclination between $35^{\circ}$ and $145^{\circ}$. The internetwork $\alpha B$ histogram is peaked between 16 and 26 Gauss, and it has to be recalled that values resulting from the inversion may eventually be compatible with weaker fields in the medium (see Sect. 2.2). However, as discussed in that section, even if the true field is roughly 10 Gauss, the azimuth is determined by the inversion. It is only when the local average field strength is lower than 5 Gauss that the uncertainty is too large for determining the azimuth.

The value of 45 Gauss that separates network and internetwork has been determined by adjusting the contour plot of a given field strength to the main features of the longitudinal field map. This value is obviously empirical. From several experiences with several different maps, the magnetic field value giving the best adjustment may vary from 45 Gauss up to
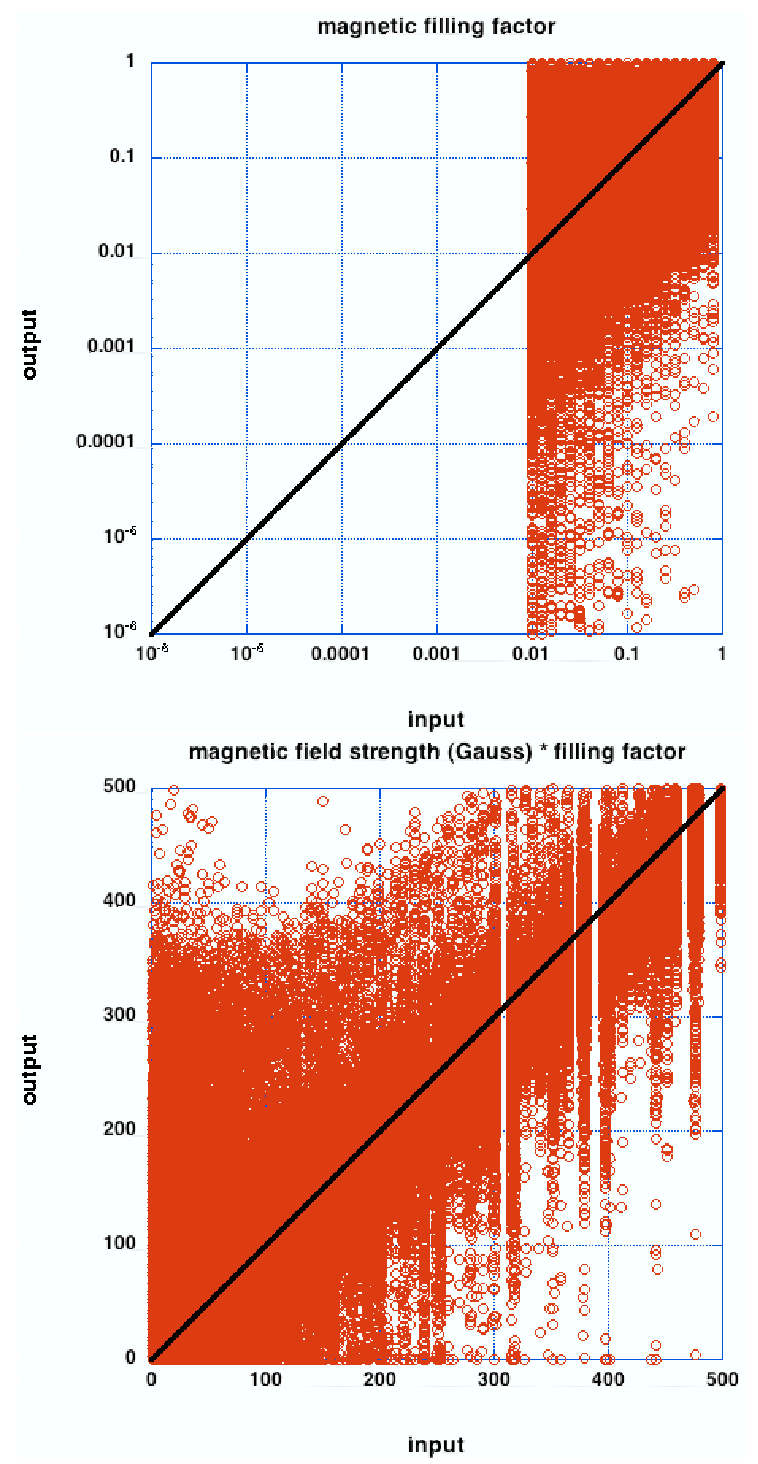

100 Gauss, depending on the map and the level of activity in it. It can be seen in Fig. 16, however, that the network and internetwork field strengths are sufficiently different that the separation value can be chosen in a wide range. In the present map, the network part displays more positive polarity pixels than negative polarity ones (the $25^{\circ}$ peak of the network inclination histogram is higher than the $155^{\circ}$ one, see Fig. 13). This is due to the particular location of the map with respect to the active region. The fact that the 45 Gauss frontier is empirical and approximate may explain why a similar trend is visible in the internetwork inclination histogram, which also displays more positive polarity pixels than negative polarity ones.

\section{Results from Fe I $6301.5 \AA$}

The heights of formation of Fe I 6302.5 and 6301.5 were derived by using the opacity calculation code built evaluating the continuum absorption coefficient as in the MALIP code of Landi Degl'Innocenti (1976), i.e. by including $\mathrm{H}^{-}$bound-free, $\mathrm{H}^{-}$freefree, neutral hydrogen atom opacity, and Rayleigh scattering on $\mathrm{H}$ atoms and Thompson scattering on free electrons. For the line-center optical-depth evaluation, atomic data were taken from Allen (1973) and partition functions from Wittmann. The 


\section{$\alpha B$ (input 6301) >= 45 NETWORK}
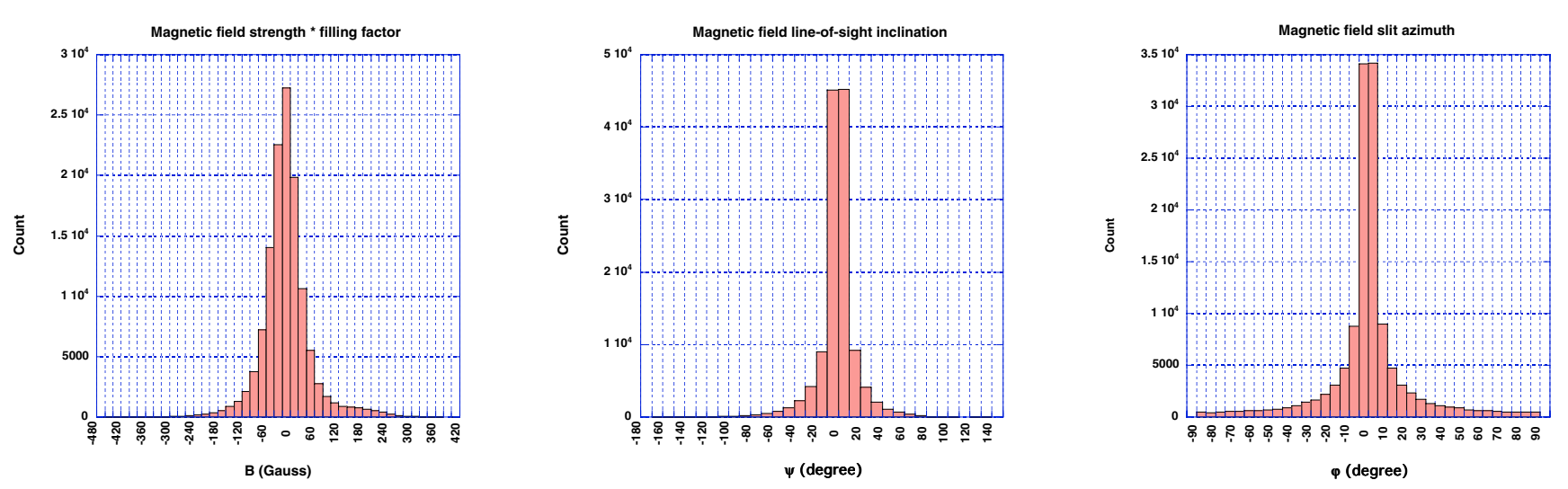

\section{$\alpha B$ (input 6301) $<45$ INTERNETWORK}

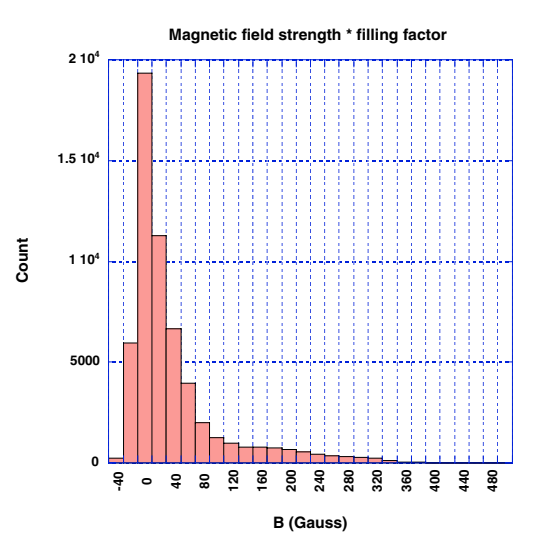

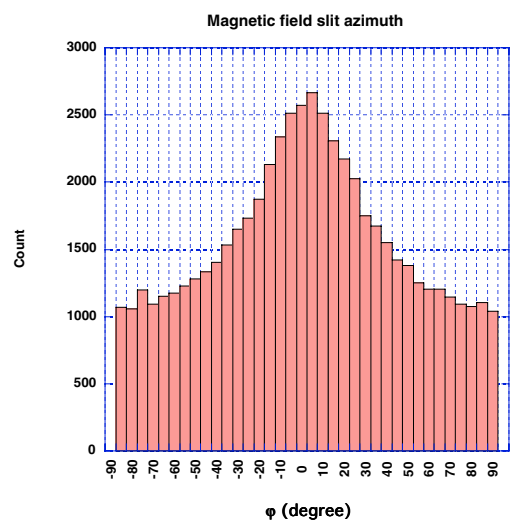

Fig. 7. Same as Fig. 5, but now for the Fe I 6301.5 line: accuracy of the UNNOFIT2 inversion.

temperature, electron pressure, and gas pressure were taken from the Maltby et al. Quiet Sun Photospheric Reference Model (Maltby et al. 1986), extrapolated downwards beyond $-70 \mathrm{~km}$ to $-450 \mathrm{~km}$ below the $\tau_{5000}=1$ level. Above $-70 \mathrm{~km}$, this model is very similar to the Quiet Sun FAL C (Fontenla et al. 1993). The temperature has been plotted in Fig. 8. A depth-independent microturbulent velocity field of $1 \mathrm{~km} \mathrm{~s}^{-1}$ was introduced. The LTE ionization equilibrium was assumed by using Saha's law to determine the ion abundances with respect to the neutral atom. Finally, departures from LTE in the ionization equilibrium were simulated by applying Saha's law with a constant "radiation temperature" of $5100 \mathrm{~K}$ instead of the electron temperature provided by the atmosphere model, for depths higher than the one corresponding to $\tau_{5000}=0.1$. The height of formation of the line center was then determined as follows: given the grid of linecenter optical depths, which provides the optical depth along the vertical as a function of height from the atmosphere model, the height of formation of the line center is the one for which the optical depth along the line of sight is unity (Eddington-Barbier approximation), i.e. the one for which $\tau / \mu=1$, where $\tau$ is the line center optical depth along the vertical, and $\mu$ is the cosine of the heliocentric angle $\theta$ (taken here as zero). By applying this method, the Fe I 6302.5 line center was found to be formed at $262 \mathrm{~km}$ and $\mathrm{Fe}$ I 6301.5 at $328 \mathrm{~km}$, thus $66 \mathrm{~km}$ higher, above the $\tau_{5000}=1$ level. These results apply to line center, whereas the inversion involves the whole profile, and the height of formation varies along the profile. In this respect, one has to be cautious with the concept of formation height (Sánchez Almeida et al. 1996). However, that 6301.5 is formed higher than 6302.5 remains true at any wavelength along the profile, so that it probably remains true for the magnetic fields resulting from the inversion.

The field vector map derived from Fe I 6301.5 (UNNOFIT2 inversion) has been plotted in Fig. 11. The comparison with the 6302.5 map of Fig. 10 shows the same network (homogeneous) and internetwork (turbulent) zones. This trend is confirmed by the histograms that are plotted in Fig. 14, for the magnetic 6302.5 local-average field strength $\alpha B_{6302}>45$ Gauss and $\alpha B_{6302}<45$ Gauss. The $6302.5 \alpha B$ values were used instead of 6301.5 , because the behavior of the map with $\alpha B$ is not the same in 6301.5 as in 6302.5. Whereas in 6302.5 the contour $\alpha B_{6302}=45$ well draws the frontier between network and internetwork (see the contour in Fig. 10), in 6301.5 

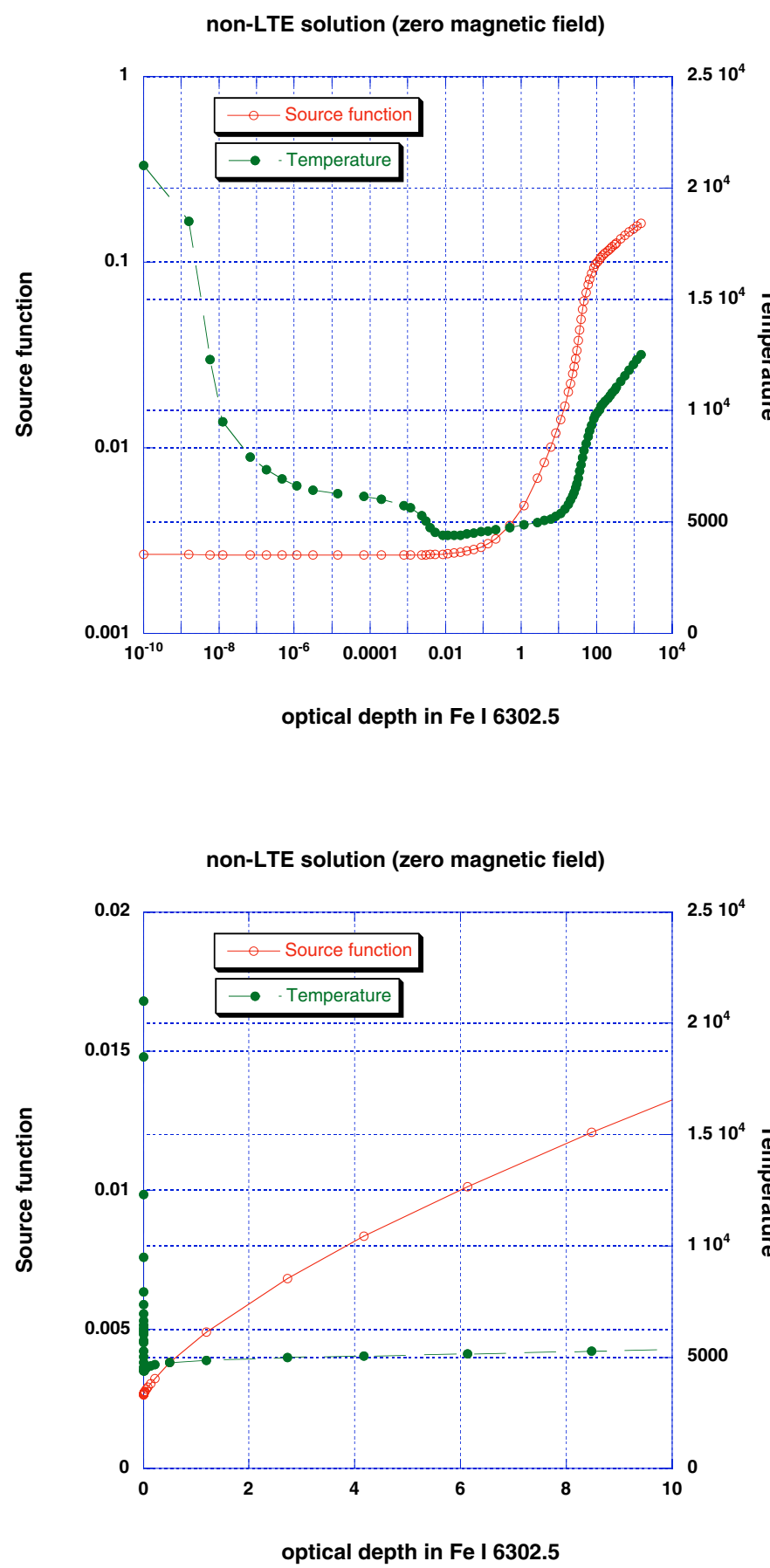

Fig. 8. Source function as a function of the line optical depth as the result of a non-LTE solution in zero magnetic field in a Quiet Sun model atmosphere. Upper figure: logarithmic scales; lower figure: linear scales, showing a linear behavior compatible with the Milne-Eddington atmosphere in the low $\tau$ region. Such a solution has been used to test the validity of the Milne-Eddington approximation used by UNNOFIT. The atmosphere temperature is plotted on the right $y$-axis. The height ranges from $-451 \mathrm{~km}$ below $\tau_{5000}=1$ (right of the figure) to $2140 \mathrm{~km}$ above $\tau_{5000}=1$ (left of the figure).

values $\alpha B_{6301}>45$ are found in both the network and internetwork (while $\alpha B_{6301}<45$ is only found in the internetwork). The trends observed in the network (upper row of Fig. 14) are comparable to the ones observed with 6302.5 (see Fig. 13): the local average field strength histogram displays the same decreasing behavior; the line-of-sight inclination angle again shows a

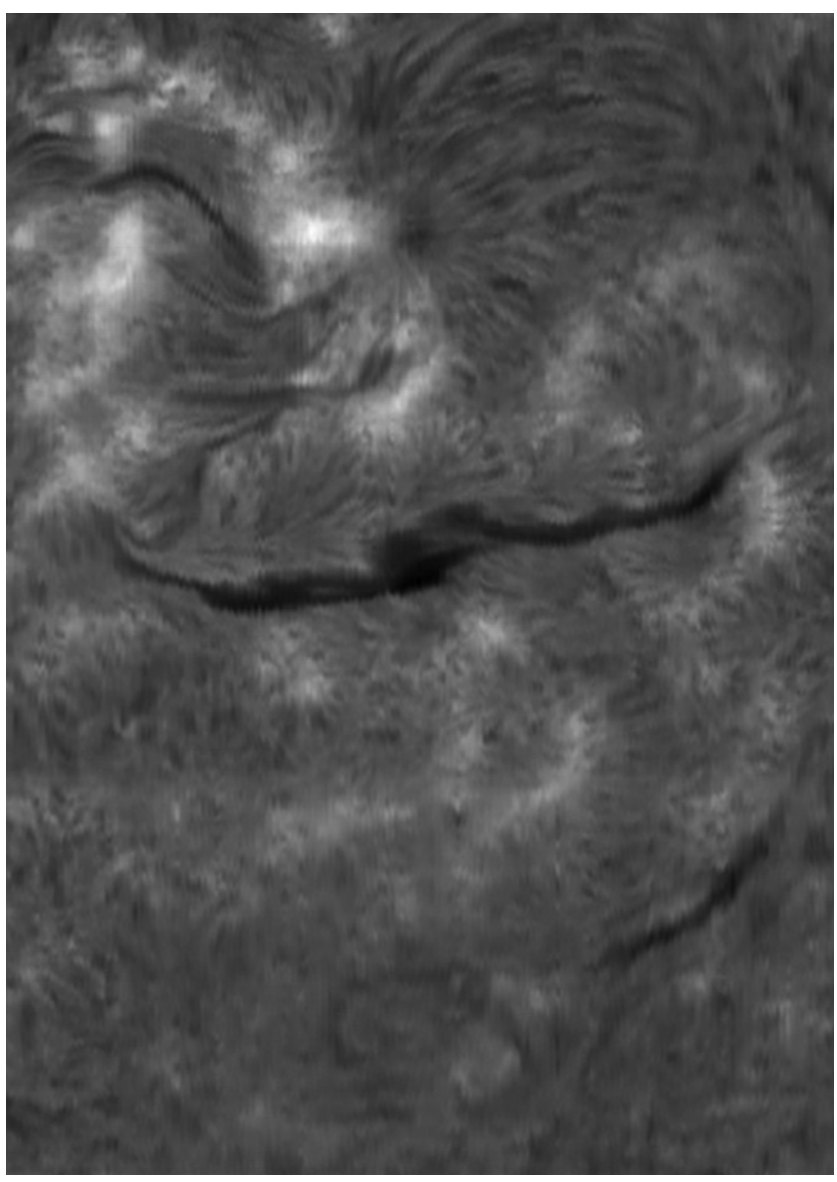

Fig. 9. H $\alpha$ THEMIS map of 7 December 2003. In the upper part: active region NOAA 517 . The scan was centered $17.5^{\circ} \mathrm{W}$ in longitude and $12.9^{\circ} \mathrm{S}$ in latitude from the disk center. The map size is $240 \times 340$ arcsec. The slit, which defines the vertical axis of the figure, was oriented Solar North.

non-horizontal trend, but spread more than with 6302.5 (the peaks that are visible in the extremities of the inclination histogram correspond to points where the inversion failed. This is more frequent with UNNOFIT2 - 6301.5, which is not a Zeeman triplet - than with UNNOFIT - 6302.5, which is a Zeeman triplet). As for the azimuth, the trend is comparable in 6301.5 and 6302.5, where a prominent peak is visible in the histogram, leading to a very homogeneous field azimuth.

The histograms of the lower row of Fig. 14, contain the internetwork values. As in 6302.5 (see Fig. 13), the azimuth histogram is nearly flat, suggesting a turbulent azimuth. As for the inclination, as in 6302.5 a horizontal trend appears with the field inclination contained between $75^{\circ}$ and $105^{\circ}$, but one has to recall that the inclination is determined within $\pm 30^{\circ}$ accuracy with 6301.5. Nevertheless, the internetwork field determined $66 \mathrm{~km}$ higher with 6301.5 appears more horizontal than the one determined lower with 6302.5. One is then led to find a turbulent (horizontal) field in the internetwork. The localaverage field-strength histogram also displays a maximum at about $\alpha B=20$ Gauss, so that the local average magnetic field strength does not seem to change with altitude. It was, however, pointed out in Sect. 2.2 that such an output of the inversion may be compatible with a weaker average local magnetic field. Again, the peaks that are visible in the extremities of the inclination histogram correspond to points where the inversion failed, which is more frequent with UNNOFIT2 than with UNNOFIT. 


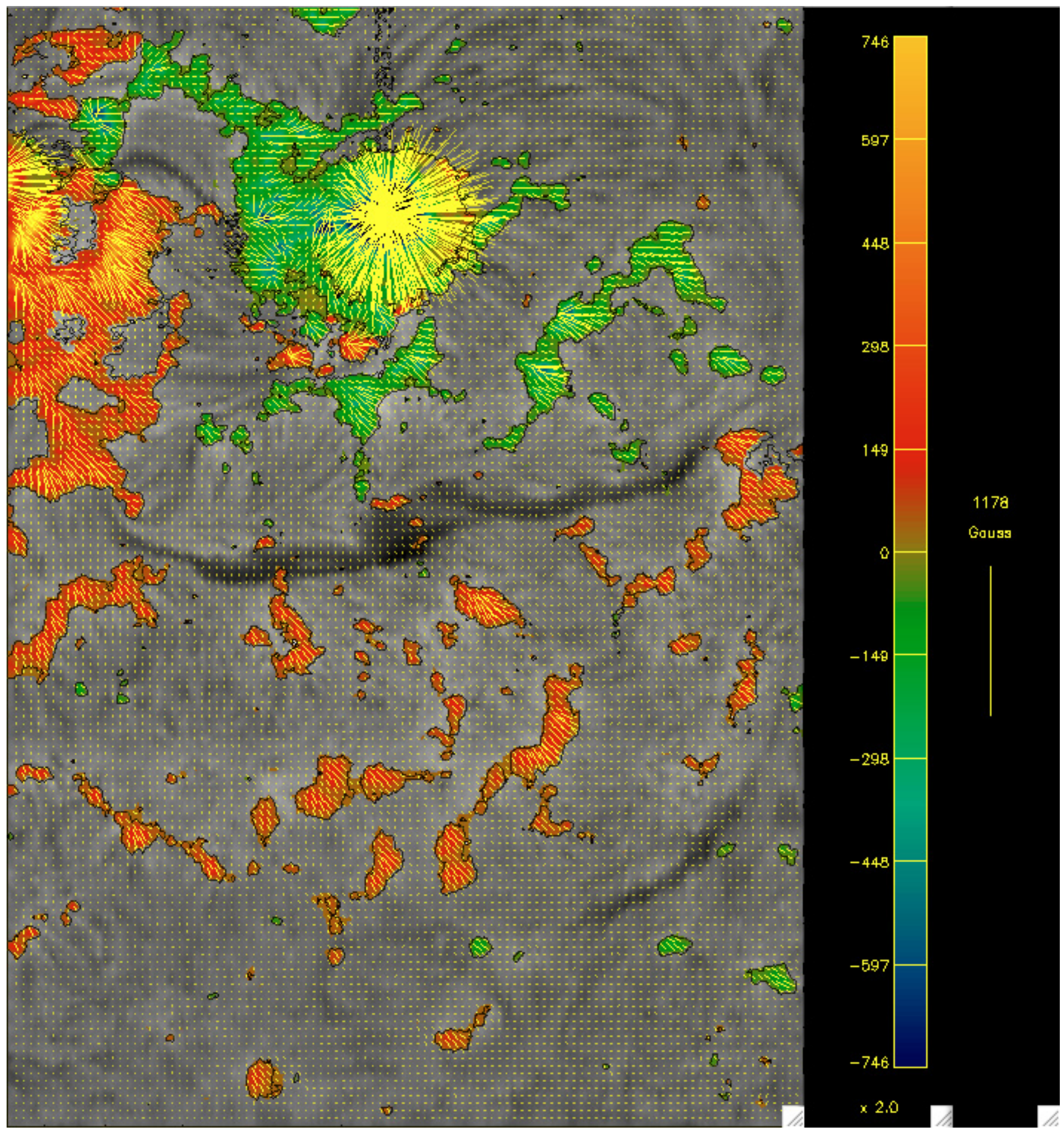

Fig. 10. NOAA 517 local average vector magnetic field map from Fe I 6302.5 A line (THEMIS 7 December 2003 observation, inverted with UNNOFIT). The longitudinal component is represented following the color scale (cold colors - blue, green - for fields entering the Sun, warm colors - red, yellow - for fields leaving the Sun). The transverse field is represented by scaled dashes, without arrow because the fundamental ambiguity is not solved. The dash scale is drawn at the right side of the figure, and its value is the largest transverse field strength of the map. The contours draw the limit $\alpha B=45$ Gauss, which is found to separate the network from the internetwork. In the internetwork the local average field is too small to be drawn, letting the ground $\mathrm{H} \alpha$ map to be visible.

\section{Comparison between the two line results}

The next step is to compare the results obtained with the two lines 6302.5 and 6301.5 , which is formed $66 \mathrm{~km}$ higher. The two lines were inverted independently. The comparison was made by plotting the histograms of the quantity differences. These histograms have been plotted separately for $\alpha B_{6302}>45$ and $\alpha_{6302}<45$ Gauss, because $\alpha B_{6302}=45$ Gauss draws the frontier well between network and internetwork (that $\alpha B_{6301}$ does not). Figure 15 displays the histograms of the differences, for the whole map separated in three thirds represented with three different colors placed in the same order from top to bottom. The 6302.5 value is subtracted from the 6301.5 one. One of the aims is to answer the question of whether the internetwork turbulence 


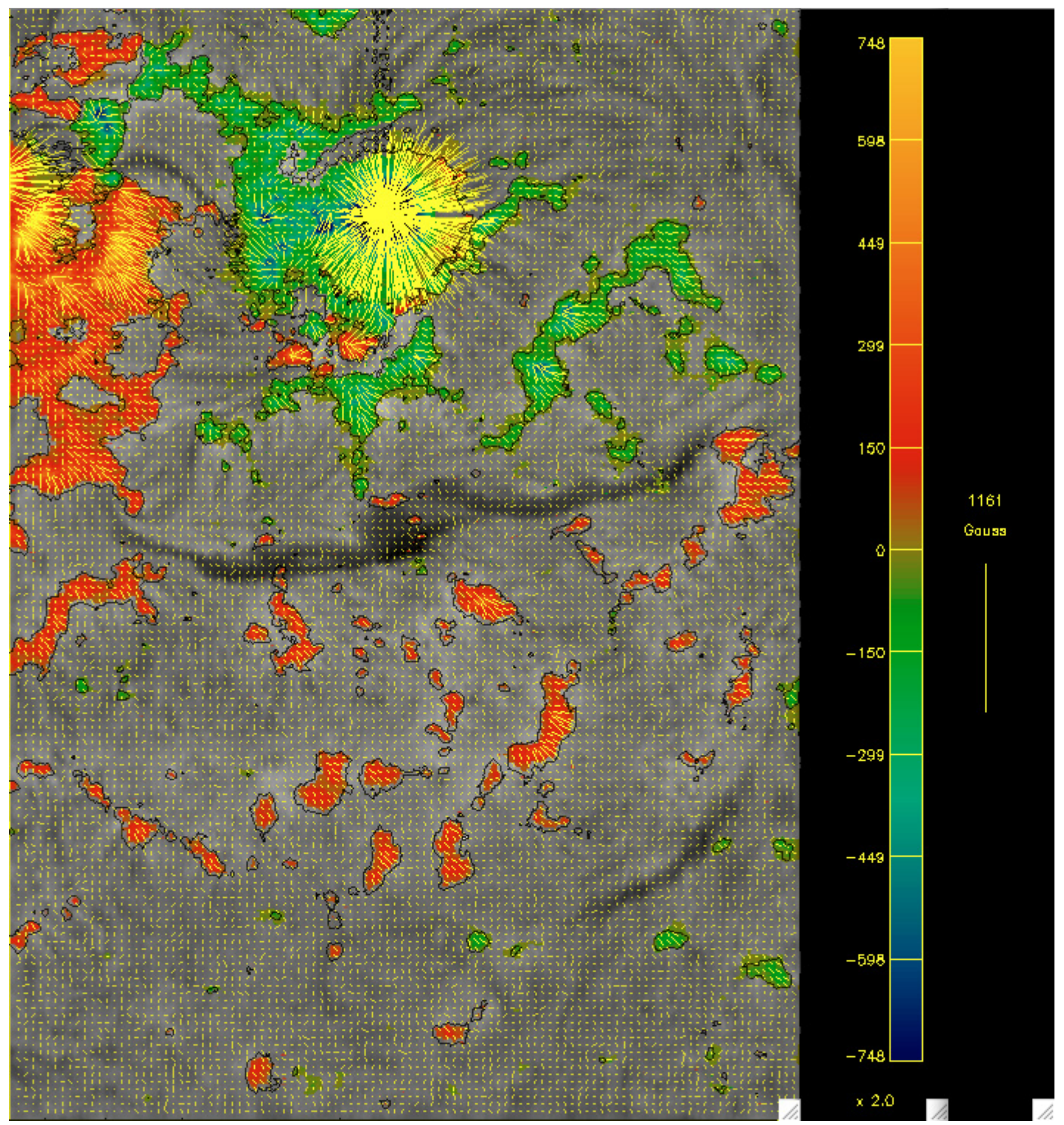

Fig. 11. NOAA 517 local average vector magnetic field map from Fe I 6301.5 A line (THEMIS 7 December 2003 observation, inverted with UNNOFIT2; same legend as Fig. 10). The contours are those derived from Fe I 6302.5.

observed is of solar origin. If this turbulence is pure detector noise, the histogram of the azimuth differences is flat because the two lines are recorded in different regions of the camera. If, on the contrary, a non-flat histogram is obtained, then by a reduction ad absurdum the turbulence is not pure noise but includes a non-negligible solar contribution. As seen below, this is the case. The Earth atmosphere effect on the line difference is expected to be small because the atmosphere acts in the same manner on the two lines. The same reasoning can be applied to the inclination, but we prefer to focus on the azimuth, because it depends (roughly) only on the linear polarization, whereas the inclination depends on both linear and circular polarizations. As the circular polarization is higher than the linear one, the accuracy is better on it and the difference in accuracy between linear and circular polarizations could eventually explain the observed horizontal trend for the inclination. As we do not discard this possibility, we focus our attention on the azimuth behavior that only involves the linear polarization.

In both network and internetwork, the local average magnetic field strength is found unchanging with altitude, and the difference histogram is peaked at 0 . 


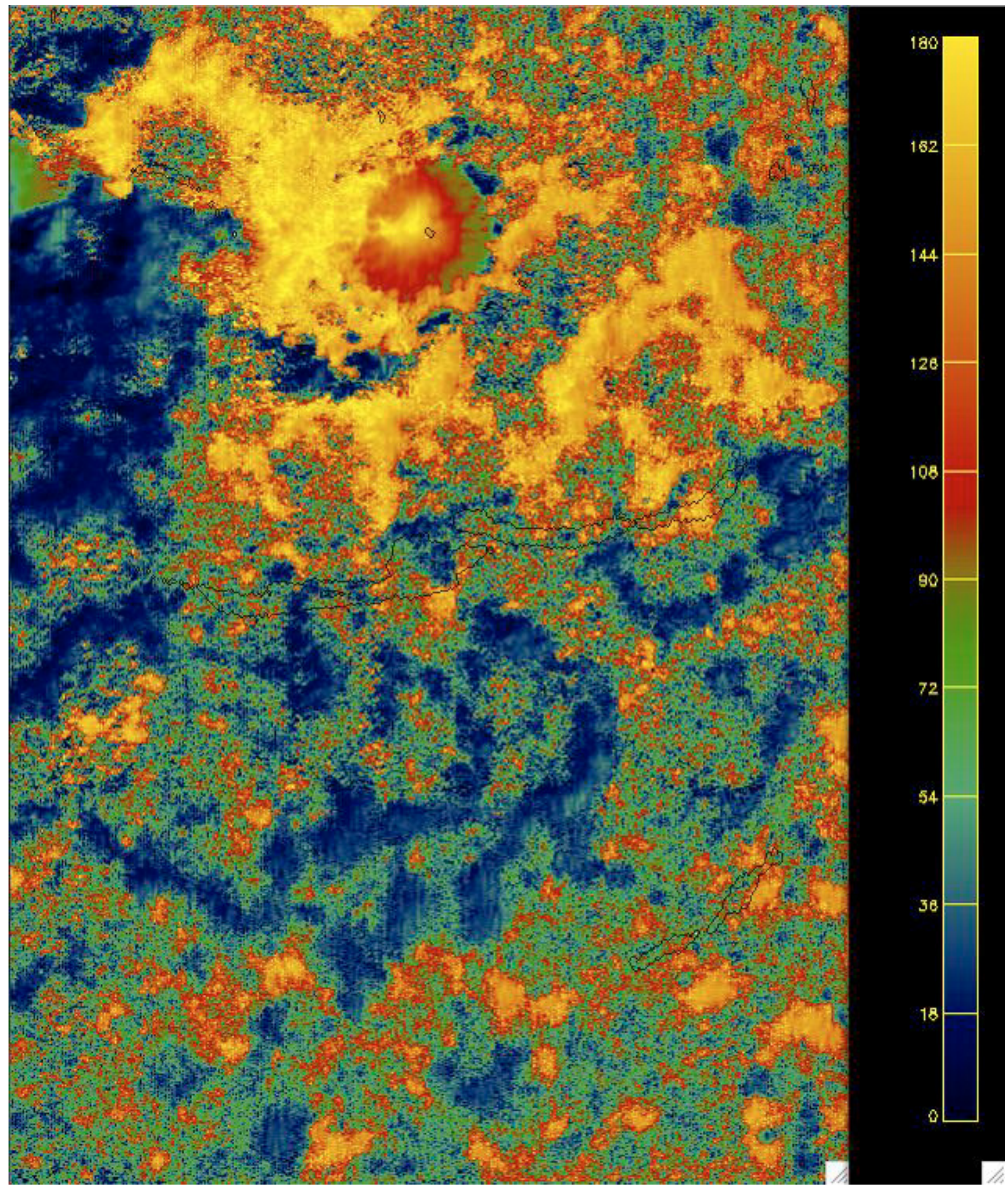

Fig. 12. Map of the magnetic field line-of-sight inclination angles of Fig. 10. The angle is the one between the magnetic field vector and the line-of-sight oriented towards the observer, from $0^{\circ}$ - blue, the field vector points towards the observer - to $180^{\circ}-$ yellow, the field vector points towards the disk center - . The contour of the $\mathrm{H} \alpha$ filament has been superimposed.

As for the field direction, let us examine the network first. The inclination shows a difference of $\pm 30^{\circ}$, which is higher than the UNNOFIT $\pm 5^{\circ}$ and UNNOFIT2 $\pm 10^{\circ}$ uncertainties (see Sect. 2.2), and corresponds to the fact that the network field is found spread more in 6301.5 than in 6302.5 , but is always non-horizontal. The difference in azimuth is $\pm 10^{\circ}$, within the same order of magnitude as the UNNOFIT and UNNOFIT2 


\section{$\alpha B(6302)>=45$ NETWORK}
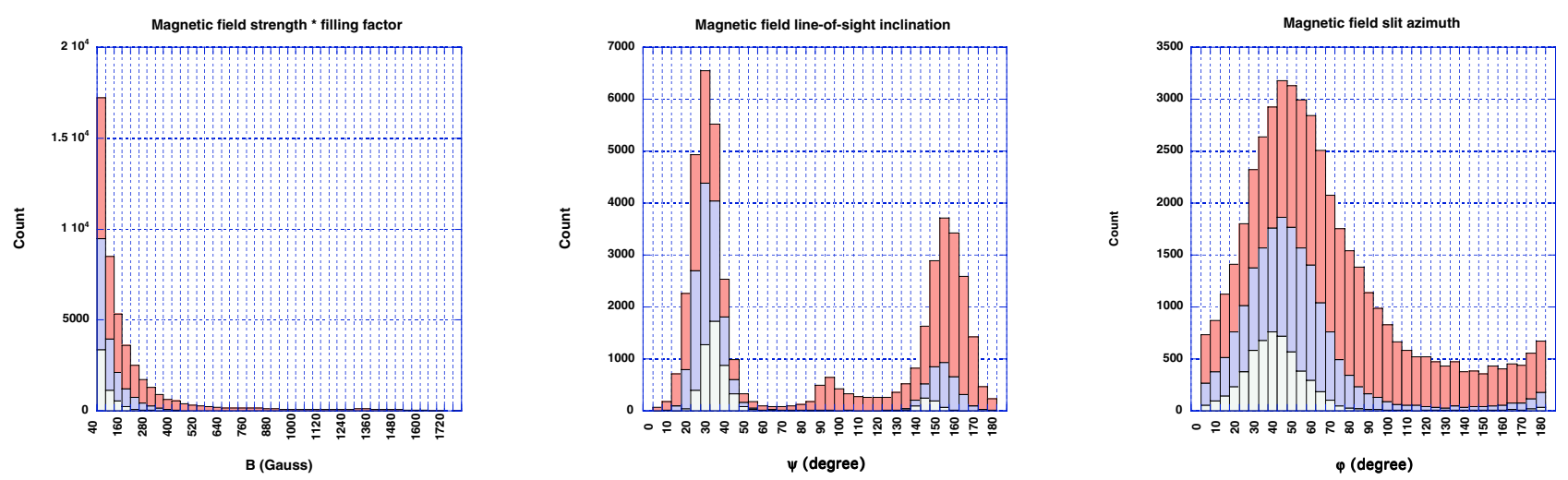

\section{$\alpha B(6302)<45$ INTERNETWORK}

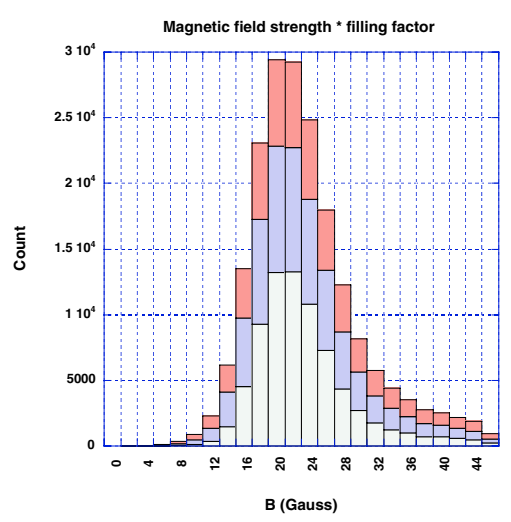

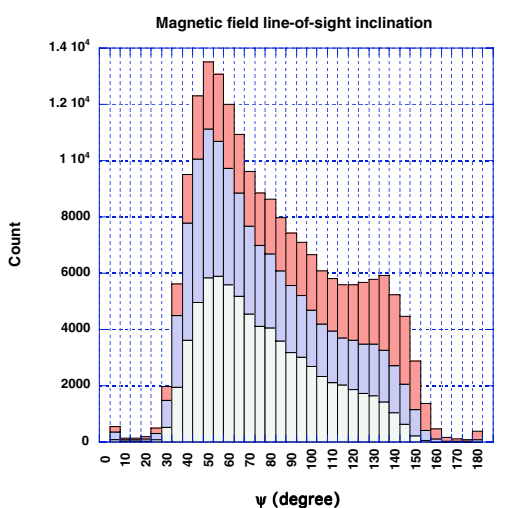

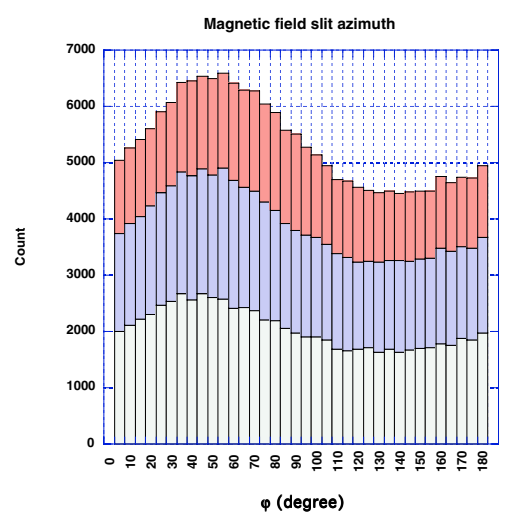

Fig. 13. Histograms of the local average magnetic field strength and direction from the Fe I $6302.5 \AA$ line, for the full NOAA 517 map divided in 3 horizontal scans stacked up in the histograms in the same order, combining pixels having either the local average magnetic field strength, which is the product of the magnetic field strength by the magnetic filling factor, larger than 45 Gauss (network field), or lower than 45 Gauss (internetwork field).

accuracies. In the network, the azimuth is homogeneous and the same at the two heights corresponding to the two lines.

As discussed above we focus our attention on the azimuth histogram for the internetwork field direction. It is not flat, with a width of $\pm 40^{\circ}$, larger than the UNNOFIT and UNNOFIT2 accuracies that have been determined, and corresponding to each of the two lines (see Sect. 2.2). That the histogram shows a non-flat shape that is larger than the accuracy leads us to conclude there is coherence between the field azimuths observed in the two lines, and then a solar origin to the observed field direction turbulence. Moreover, that a coherence is found between the azimuths determined from both lines discards the idea that the local average field strength would be smaller than the one resulting from the inversion, a possibility that was outlined in Sect. 2.2, because in the case of fields strengths smaller than 5 Gauss in Fe I 6302.5 and 10 Gauss in Fe I 6301.5 (the highest value being the one to be retained for the line comparison), the azimuth is indeterminate and remains noisy. We therefore determine an about 20 Gauss turbulent field in the internetwork region.

\section{Conclusion}

We have performed UNNOFIT inversion on spectropolarimetric data obtained for Fe I 6302.5 and 6301.5 on 7 December 2003 , in a region including one and a half sunspot, plages, a filament, and a quieter region, localized near the disk center. UNNOFIT is an inversion code (Landolfi et al. 1984) that includes the magneto-optical and damping effects (Landolfi \& Landi Degl'Innocenti, 1982) and that is based on the Marquardt algorithm applied to the Unno-Rachkowsky solution for the Stokes parameters emerging from a Milne-Eddington atmosphere. In the present work, UNNOFIT was completed by introducing a two-component atmosphere, having a magnetic component and a non-magnetic component, the other physical parameters being the same in both components. The inversion was performed separately on each line. Concerning the UNNOFIT accuracy, our tests show that in fact it is not possible to determine the magnetic field strength $B$ and the magnetic filling factor $\alpha$ separately, but that their product $\alpha B$, which 

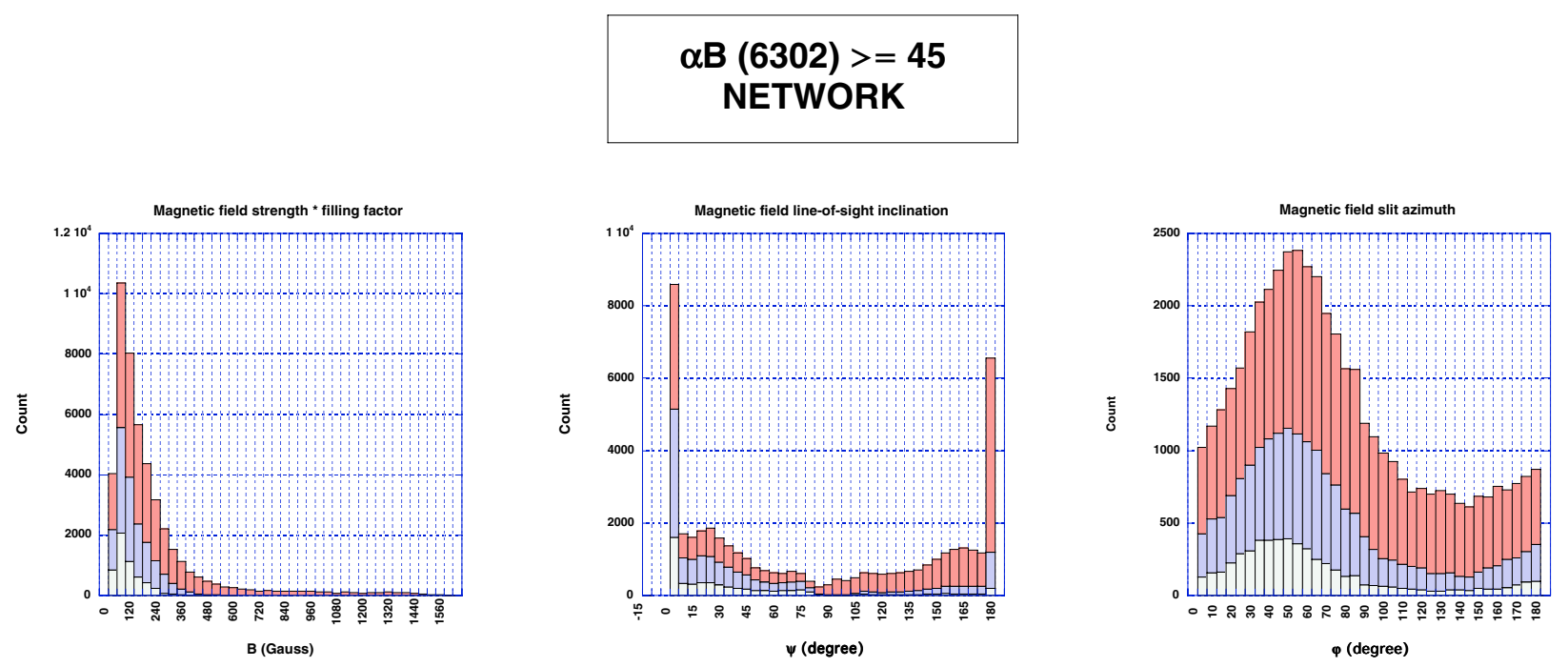

\section{$\alpha B(6302)<45$
INTERNETWORK}
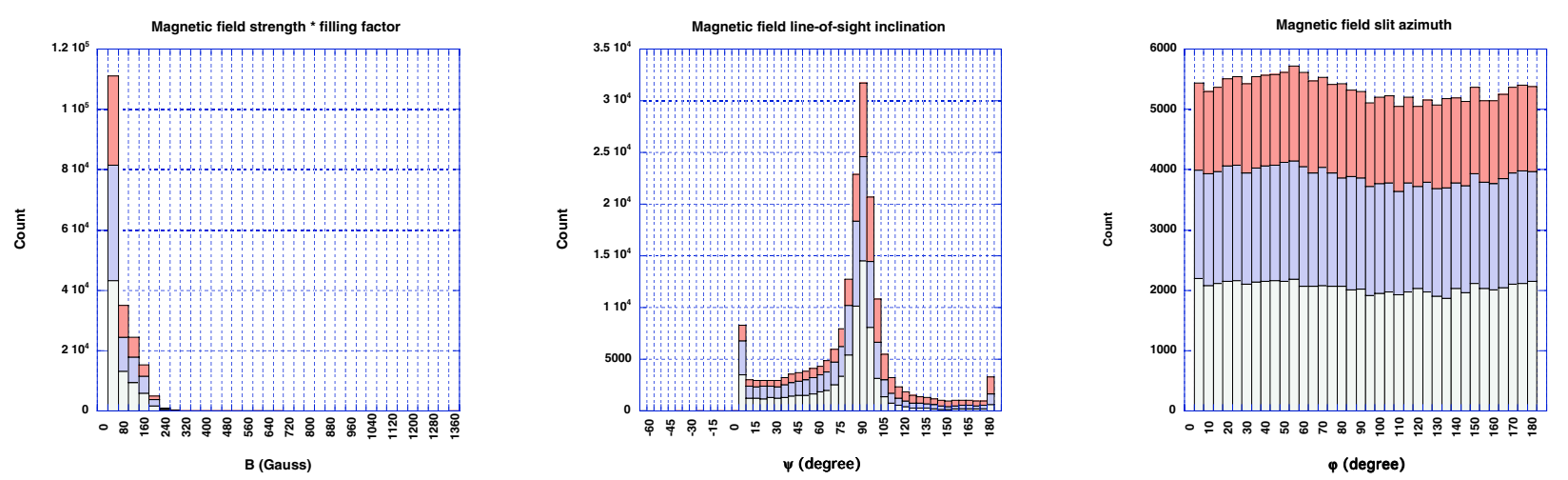

Fig. 14. Histograms of the local average magnetic field strength and direction from the Fe I $6301.5 \AA$ line, for the full NOAA 517 map divided in 3 horizontal scans stacked up in the histograms in the same order, combining pixels having either the local average magnetic field strength, which is the product of the magnetic field strength by the magnetic filling factor, larger than 45 Gauss in Fe I $6302.5 \AA$ (network field), or lower than 45 Gauss in Fe I $6302.5 \AA$ (internetwork field).

is the local average magnetic field (the average magnetic field in each point), is determined by the inversion. The magnetic flux is only the longitudinal component of the local average magnetic field. The maximum of our $\alpha B$ histograms is about $\alpha B=20$ Gauss, localized in the internetwork, which is compatible with the field strength $1 \mathrm{kGauss}$ filling $2 \%$ of space as indicated by several authors, as stated in the introduction. This maximum is the same in the two lines, although 6301.5 is formed $66 \mathrm{~km}$ higher than 6302.5. Although the histogram maximum at 20 Gauss could eventually be due to an effect of the inversion and be not incompatible with weaker local average magnetic field strengths, the observed coherence of the 6302.5 and 6301.5 determined azimuths discards the idea of a weak field, because in this case the azimuth would be indeterminate and would remain noisy. Therefore we determine around 20 Gauss local average strength for the internetwork field.

We show that the value $\alpha B_{6302}=45$ Gauss separates the network $\left(\alpha B_{6302}>45\right)$ from the internetwork $\left(\alpha B_{6302}<45\right)$. The local average magnetic field is then stronger in the network than in the internetwork, as can be seen in Fig. 16, which is a
3D plot of the local average magnetic field of the NOAA 517 region on 7 December 2003, where the internetwork is a sort of 20 Gauss ground on which the network emerges.

The main interest of the present work is to provide results for the field direction, a problem that has not been widely explored. Such a purpose can only be reached with a 4-Stokes parameter analysis, which is also not very frequent.

As for the field direction, in the network the field is found to be rather vertical (spread more at higher altitudes), and it has a homogeneous direction in inclination and azimuth. In the internetwork, the field is found to be turbulent in direction, with a horizontal trend (spread more at lower altitudes).

As the internetwork pixels are usually considered as too noisy to give a significant result from the inversion, we have compared the results issued from the 6302.5 and 6301.5 inversion and, in particular, the azimuth of the turbulent 20 Gauss internetwork field. We show that the azimuths independently obtained for 6302.5 and 6301.5, though turbulent, are coherent, so that we conclude there is solar nature to this turbulence, until someone can find another explanation of the non-flat behavior of 

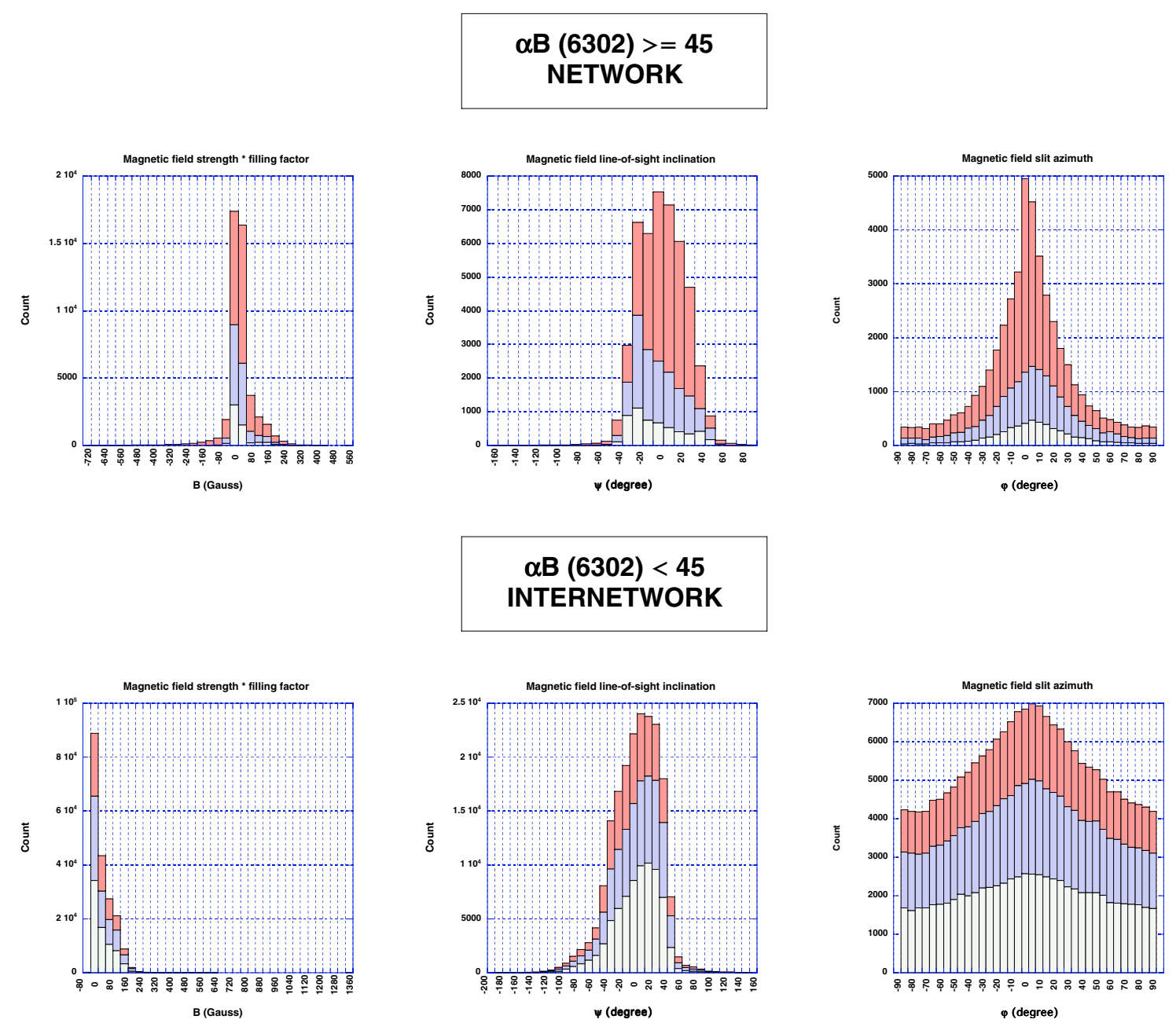

Fig. 15. Histograms of the differences in the Fe I $6301.5 \AA$ results and Fe I $6302.5 \AA$ results for the full NOAA 517 map divided in 3 horizontal scans stacked up in the histograms in the same order and combining pixels having either the local average magnetic field strength, which is the product of the magnetic field strength by the magnetic filling factor, larger than 45 Gauss in Fe I $6302.5 \AA$ (network field), or lower than 45 Gauss in Fe I $6302.5 \AA$ (internetwork field).

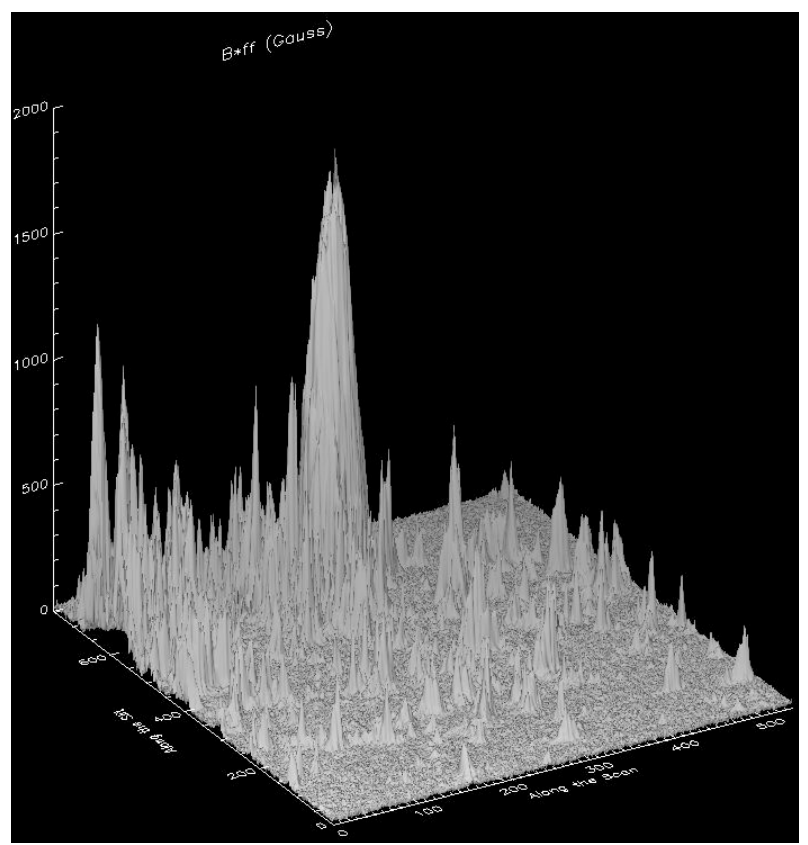

Fig. 16. 3D plot of the local average magnetic field strength through the Fe I $6302.5 \AA$ map of Fig. 10. The internetwork is a sort of 20 Gauss ground on which the network emerges. the azimuth difference histogram that we observed (see Fig. 15, lower right figure), to refute this conclusion.

Acknowledgements. The authors are deeply indebted to the anonymous referee for helpful comments and suggestions.

\section{References}

Allen, C. W. 1973, Astrophysical Quantities, 3rd ed., Univ. of London, the Athlone Press.

Arnaud, J., Mein, P., \& Rayrole, J. 1998, Proceedings of A Crossroads for European Solar \& Heliospheric Physics, Tenerife, ESA SP-417, 213

Auer, L. H., Heasley, J. N., \& House, L. L. 1977, Sol. Phys., 55, 47

Bellot Rubio, L. R., \& Collados, M. 2003, A\&A, 406, 357

Bommier, V., \& Landi Degl'Innocenti, E. 1996, Sol. Phys., 164, 117 (also SPW1)

Bommier, V., \& Rayrole, J. 2002, A\&A, 381, 227

Bommier, V., Derouich, M., LandiDegl'Innocenti, E., Molodij, G., \& Sahal-Bréchot, S. 2005a, A\&A, 432, 295

Bommier, V., Rayrole, J., \& Eff-Darwich, A. 2005b, A\&A 435, 1115

Dominguez Cerdeña, I., SánchezAlmeida, J., \& Kneer, F. 2003, A\&A, 407, 741

Faurobert, M., Arnaud, J., Vigneau, J., \& Frisch, H. 2001, A\&A, 378, 627

Fontenla, J. M., Avrett, E. H., \& Loeser, R. 1993, ApJ, 406, 319

Grossmann-Doerth, U., Keller, C. U., \& Schüssler, M. 1996, A\&A 315, 610

Harvey, J., Livingston, W., \& Slaughter, C. 1972, in Line Formation in the Presence of Magnetic Fields, High AltitudeObservatory, NCAR, Boulder, Colorado, 227 
Keller, C. U., Deubner, F. L., Egger, U., Fleck, B., \& Povel, H. P. 1994, A\&A, 286, 626

Khomenko, E. V., Collados, M., Solanki, S. K., Lagg, A., \& Trujillo Bueno, J. 2003, A\&A, 408, 1115

Landi Degl'Innocenti, E. 1976, A\&AS, 25, 379

Landi Degl'Innocenti, E., Bommier, V., \& Sahal-Bréchot, S. 1991a, A\&A, 244, 391

Landi Degl'Innocenti, E., Bommier, V., \& Sahal-Bréchot, S. 1991b, A\&A, 244, 401

Landi Degl'Innocenti, E., \& Landolfi, M. 2004, Polarization in Spectral Lines (Kluwer Acad. Publ.)

Landolfi, M., \& Landi Degl'Innocenti, E. 1982, Sol. Phys., 78, 355

Landolfi, M., Landi Degl'Innocenti, E., \& Arena, P. 1984, Sol. Phys., 93, 269

Lin, H. 1995, ApJ, 446, 421

Lin, H., \& Rimmele, T. 1999, ApJ, 514, 448

Lites, B. W. 2002, ApJ, 573, 431

Lites, B. W., \& Skumanich, A. 1990, ApJ, 348, 747

Lites, B. W., \& Socas Navarro, H. 2004, ApJ, 613, 600
Maltby, P., Avrett, E. H., Carlsson, M., et al. 1986, ApJ, 306, 284

Rees, D. E. 1969, Sol. Phys., 10, 268

Ruiz Cobo, B., \& del Toro Iniesta, J. C. 1992, ApJ, 398, 375

Sánchez Almeida, J. 1997, ApJ, 491, 993

Sánchez Almeida, J., \& Lites, B. W. 2000, ApJ, 532, 1215

Sánchez Almeida, J., Ruiz Cobo, B., \& delToro Iniesta J. C. 1996, A\&A, 314, 295

Sánchez Almeida, J., DominguezCerdeña, I., \& Kneer, F. 2003, ApJ, 597, L177 Skumanich, A., \& Lites, B. W. 1987, ApJ, 322, 473

Socas-Navarro, H., \& SánchezAlmeida, J. 2002, ApJ, 565, 1323

Socas-Navarro, H., \& Lites, B. W. 2004, ApJ, 616, 587

Stenflo, J. O. 1973, Sol. Phys., 32, 41

Stenflo, J. O. 1982, Sol. Phys., 80, 209

Trujillo Bueno, J., \& LandiDegl'Innocenti, E. 1996, Sol. Phys., also Solar Polarization,Proceedings of an International Workshop held in St petersburg, Russia, 8-12 May, 1995, ed. J. O. Stenflo, \& K. N. Nagendra, 164, 135

Trujillo Bueno, J., Shchukina, N., \& Asensio Ramos, A. 2004, Nature, 430, 326 